\begin{tabular}{|l|l|c|l|}
\hline Eiszeitalter u. Gegenwart & 32 & $\begin{array}{c}137-161 \\
\text { 7 Abb., 1 Tab., 2 Taf. }\end{array}$ & Hannover 1982 \\
\hline
\end{tabular}

\title{
Zur Gliederung der Rißeiszeit im östlichen Rheingletschergebiet (Baden-Württemberg)
}

\author{
Albert Schreiner \& Thomas HaAg *
}

Subdivision, Riss Glacial, borehole section, morain, different weathering depth, morphology, pebble analysis, gravel, paleosol.

Bavarian Plateau, Eastern Rhine Glacier Area, Baden-Württemberg, TK 25 Nr.: 7824, 7825, 7925

Kurzfassung: Die Altmoräne des Rheingletschergebiets östlich von Biberach kann folgendermaßen gegliedert werden (von außen nach innen):

$\mathrm{M}$ in d e $1 \mathrm{~m}$ or ä n e (M), sehr flache, mehr als $4 \mathrm{~m}$ tief verwitterte Moränenwälle.

A 1 te res Riß (= Zungenriß, Rz), Kiese, Kiesmoräne und Geschiebemergeldecken mit geringer Verwitterungstiefe $(<3 \mathrm{~m})$, die $\mathrm{zungenf}$ örmig in die meist höher liegenden Mindelmoränen vorstoßen. Trennung von $\mathrm{M}$ und $\mathrm{Rz}$ durch fossile Böden.

Mittleres Riß (= Doppelwall-Riß, Rd). Zwei deutliche, kiesige Endmoränenwälle mit geringer Verwitterung.

Die Moränen von Rz und Rd sind im Rißtal mit den mächtigen Kiesen der Typlokalität Riß zu verknüpfen.

Jüngeres Riß (nicht näher untersucht). „Jungriß“-Terrasse im Riß- und Donautal und $\mathrm{v}$ i e ll e i ch t dazugehörende Moränenwälle 4 bis $6 \mathrm{~km}$ innerhalb von Rd.

Die unterschiedliche Verwitterungstiefe zwischen Mindel- und Rißmoränen wurde durch Bohrungen auf allseitig abfallenden Kuppen ermittelt. Geröllanalysen und fossile Böden unterstützen die Gliederung.

\section{[On the Subdivision of the Riss Glacial Period in the Eastern Part of the Rhine Glacier Area (Baden-Württemberg) ]}

Abstract: The "Alt-Moräne" of the Rhine glacier area in the east of Biberach can be divided as follows (from outside to inside):

$\mathrm{M}$ indel $\mathrm{mora}$ in e (M): Very flat moraine ramparts, weathering depth more than $4 \mathrm{~m}$.

L ow e $\mathrm{r}$ R is s (= Tongue Riss, Rz). Gravel, gravelly moraine and boulder clay plains with minor weathering depth $(<3 \mathrm{~m})$, situated as tongues between collins of mostly higher elevated Mindel moraines. Separation of $\mathrm{M}$ and $\mathrm{Rz}$ by fossil soils.

$\mathrm{Middle} \mathrm{R}$ is s (= double rampart Riss, $\mathrm{Rd}$ ). Two distinct gravelly ramparts with minor weathering inside of Rz.

The moraines of $\mathrm{Rz}$ and $\mathrm{Rd}$ are linked with the thick gravels of the Riss type locality of Riss in the Riss valley.

U p p e r R is s (not investigated particularly). Upper Riss terrace in the valleys of Riss and Donau perhaps belonging to moraines inside of $\mathrm{Rd}$.

The different weathering depth of Mindel- and Riss moraines had been investigated by borings on the top of flat moraine ramparts sloping on all sides. The subdivision was supported by investigation of petrographical gravel composition and of fossil soils.

\section{[La division de la période glaciale de Riss dans la région du glacier oriental du Rhin (Baden-Württemberg) ]}

Rés u mé : La moraine ancienne (Altmoräne) de la région du glacier du Rhin de l'est de Biberach on peut divisée en parties differentes (da la péripherie au centre):

*) Anschriften der Autoren: Dr. A. S c h r e i n e r, Geologisches Landesamt Baden-Württemberg, Albertstr. 5, 7800 Freiburg i. Br.

Dr. Thomas H a a g, Institut Pflanzenern. u. Bodenkde., Bundesallee 50, 3300 Braunschweig. 
Morain e de Mindel (M): Des remparts morainiques très plats; altérés de plus de $4 \mathrm{~m}$.

$\mathrm{R}$ is s a n cien (= Riss de langue, $\mathrm{Rz}$ ). Des graviers, des moraines graveleuses et des argiles de blocaux d'altération moindre $(<3 \mathrm{~m})$, situés en langues dans les collines morainiques de Mindel d'altitude plus haute. Séparation de Mindel et de Riss par des paléosols.

$\mathrm{R}$ is s moyen (= Riss du rempart double, $\mathrm{Rd}$ ). Deux remparts morainiques graveleux bien visibles et d'altération moindre.

Les moraines de $\mathrm{Rz}$ et $\mathrm{Rd}$ sont à liér avec les graviers très épais de la localité type Riss dans la vallée de la Riss.

$\mathrm{R}$ is s plus jeun e (pas encore examiné de plus près). Une terrasse de gravier plus basse que Rd dans les vallées de la Riss et du Danube et des moraines eventuellement liés à celle-ci 4 à $6 \mathrm{~km}$ à l'intérieur du Rd.

L'altération differente entre les Moraines du Mindel et la Riss à été établie à l'aide de sondages sur les sommets de remparts penchés de tous côtés. La division est confirmée par des analyses de la composition pétrographique du gravier et par examen de les paléosols.

\section{Inhaltsverzeichnis}

1. Einleitung . . . . . . . . . . . . . . . . . . . . . . . 138

2. Das Pleistozän bei Rottum

3. Verwitterungstiefe auf Riß- und Mindelmoräne . . . . . . . . . . . . . . . . . . . . . . 142

4. Das Zungenriß $(\mathrm{Rz})$. . . . . . . . . . . . . . . . . . . . . . . . . . . . . 144

4.1. Rottumzunge . . . . . . . . . . . . . . . . . . . . . . . 145

4.2. Dürnachzunge . . . . . . . . . . . . . . . . . . . . . . 145

4.3. Rißtalzunge . . . . . . . . . . . . . . . . . . . . . . . . . . . 147

5. Ablauf der Ereignisse im Rißtal und Vergleiche . . . . . . . . . . . . . . . . . . . . . 150

6. Geröllanalysen . . . . . . . . . . . . . . . . . . . . . . . . 150

7. Untersuchungen an fossilen Böden . . . . . . . . . . . . . . . . . . . . . . 152

8. Bohrungen zur Bestimmung der Verwitterungstiefe . . . . . . . . . . . . . . . . 157

9. Aufschlußverzeichnis . . . . . . . . . . . . . . . . . . . . . . . . . 160

10. Schriftenverzeichnis . . . . . . . . . . . . . . . . . . . . . . . 160

\section{Einleitung}

Nach der Revision der pleistozänen Stratigraphie im Gebiet des Rheingletschers durch Graul (1962) und Schädel \& Werner (1963) wurde die Auffassung von der Ausdehnung der Mindel- und der Rißeiszeit im wesentlichen wieder auf PENCK \& BRÜCKNER (1909: 396, Karte) zurückgeführt. Das heißt: Die äußersten Moränen auf der Linie Warthausen-Ochsenhausen-Hauerz sind mindeleiszeitlich; die rißeiszeitlichen Moränen sind im wesentlichen auf den Riß-Doppelwall etwa $5 \mathrm{~km}$ innerhalb der Mindel-Endmoräne beschränkt. Im einzelnen verblieben jedoch Unstimmigkeiten, die sich an der verschiedenen Zuordnung der Schichten in der Kiesgrube A 7 beim Hof Riedwanger 0,6 km nördlich Rottum deutlich machen lassen. Graul (1962: 264) stellt den hier aufgeschlossenen fossilen Boden in die Zeit zwischen Günz und Mindel, die Hangendmoräne also in die Mindeleiszeit. SCHÄDEL \& WERNER (1963: 22) stellen jedoch den fossilen Boden von Riedwanger in die Zeit zwischen Mindel und Riß und damit die Hangendmoräne in die Rißeiszeit.

Schädel \& Werner (1963) fanden also, daß es im Gegensatz zu Graul (1962) im östlichen Rheingletschergebiet rißeiszeitliche Moränen gibt, die weit außerhalb des Riß-Doppelwalls liegen.

Es war das Ziel unserer Untersuchungen, der weitesten Ausdehnung der rißeiszeitlichen Vergletscherung und einer Gliederung der Rißeiszeit nachzuspüren, um damit eine Grundlage für eine neue geokartographische Darstellung des Pleistozäns in Oberschwaben zu gewinnen.

Herrn Dr. EBEL danken wir für seine Mitarbeit bei einigen Bohrungen. 


\section{Das Pleistozän bei Rottum (Abb. 2)}

Die bei Rottum, $4 \mathrm{~km}$ SW Ochsenhausen, relativ gut aufgeschlossenen und schon mehrfach untersuchten Schotter, Moränen und fossilen Böden können als Schlüsselstelle der hier behandelten Fragen betrachtet werden (Lageangaben in Abb. 2).

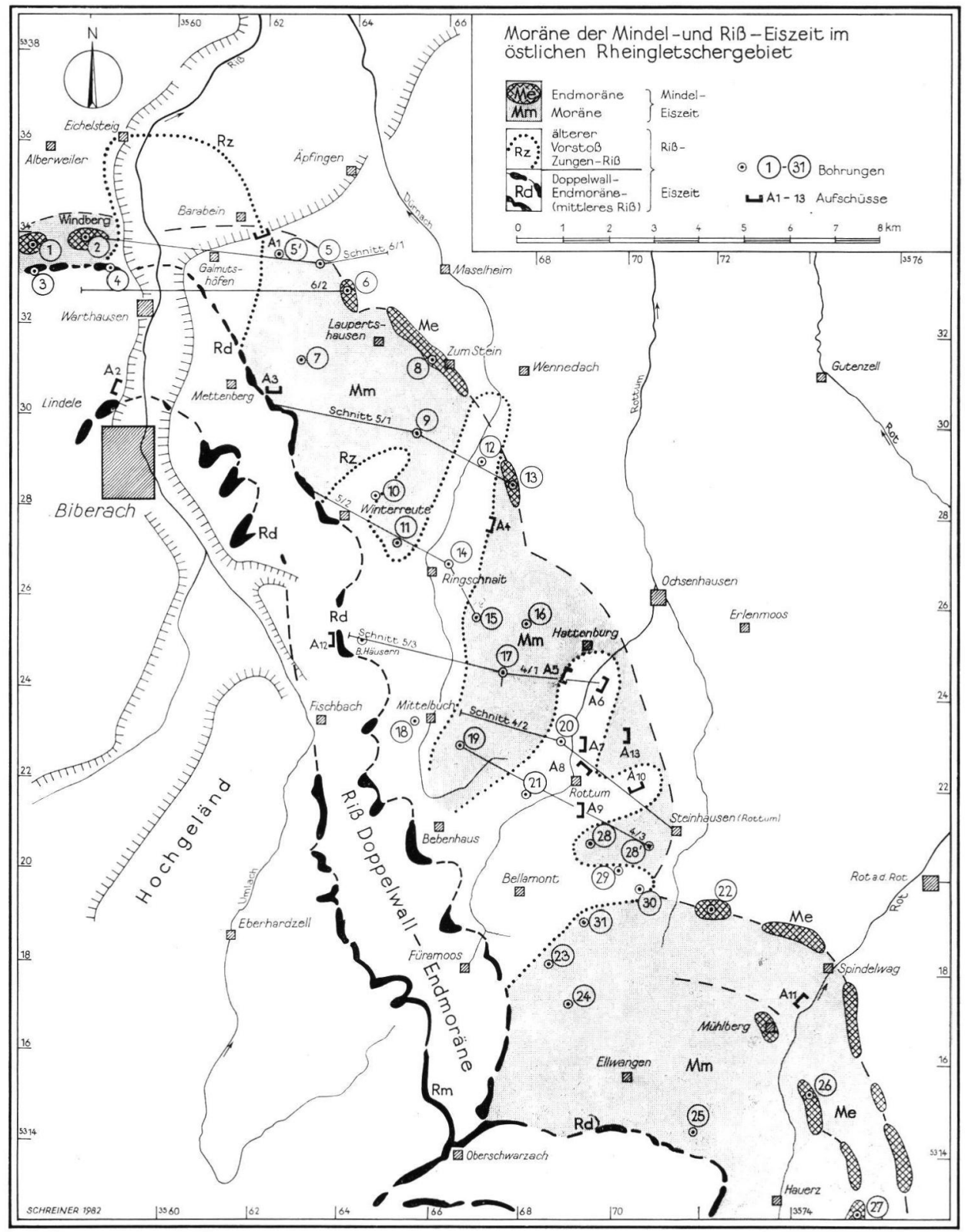

Abb. 1: Moränen der Mindel- und Rißeiszeit im östlichen Rheingletschergebiet. Mit Lage der Querschnitte Abb. 4, 5 und 6. 
WeIdenBach (1935) kartierte hier an mehreren Stellen einen fossilen Boden zwischen einem liegenden, kristallinarmen Schotter und kristallinreichen Schottern und Moränen im Hangenden. SCHÄDEL \& WeRnER (1963: 21) fanden darüber hinaus beim $\mathrm{H}$ of $\mathrm{R}$ i e d w a n g e r (A 7) einen weiteren fossilen Boden, der die kristallinreichen Schotter und Moränen unterteilt. Nach ihrer Deutung sind die bei Rottum gefundenen 3 eiszeitlichen Bildungen von oben nach unten der Riß-, Mindel- und Günz-Eiszeit zuzuordnen. Nach Graul (1962: 264), der die oberste Moräne für Mindel hält, folgen darunter Günz und Donau. Diese verschiedenen stratigraphischen Auffassungen sind von weitreichenden Folgen für die Zuordnung der Moränen und Schotter im ganzen östlichen Rheingletschergebiet.

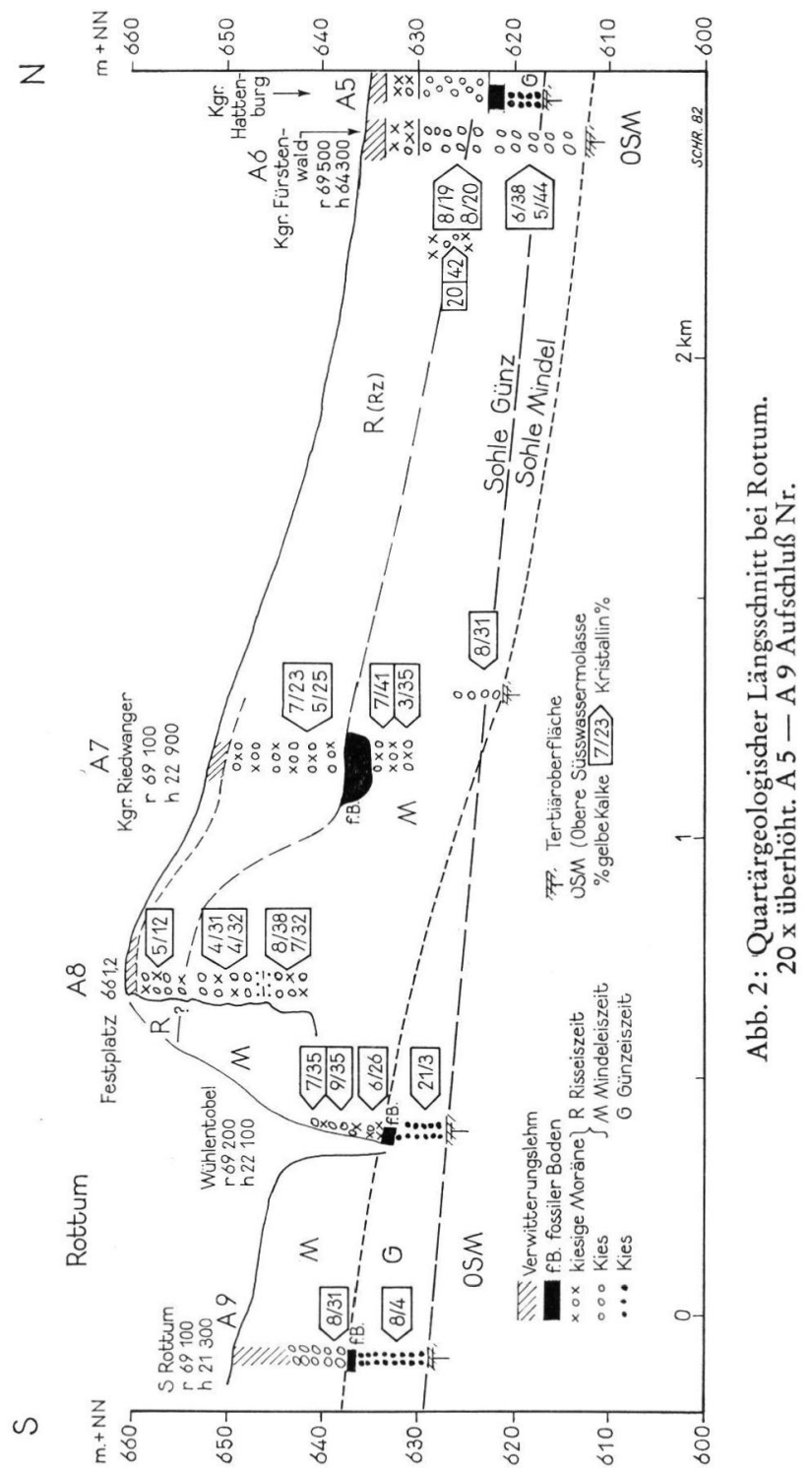


Nach unseren neuerlichen Untersuchungen ergibt sich das in Abb. 2 dargestellte Bild: Die obere Schicht sind grobkiesige, meist geschichtete, schlecht sortierte Moränen mit schlecht gerundeten, zum Teil gekritzten Geröllen. Der Kies ist weitgehend zu Nagelfluh verfestigt. Die Verwitterungstiefe (= Entkalkungstiefe) ist am Festplatz und bei der Kiesgrube Riedwanger, wo die Aufschlüsse bis auf die ebene oder fast ebene Hochfläche reichen, nur 1,5 bis 2,5 m. Die Geröllzusammensetzung dieser oberen Kiesmoräne ist durch mittleren Kristallingehalt (12 bis $25 \%$ ) gekennzeichnet. Damit vergleichbare Bildungen sind weiter im SW bei Bellamont und nach NE bis zu den Kiesgruben bei $\mathrm{H}$ a t t e $\mathrm{n}$ burg (A 5) und im Fürstenwald (A 6) als obere Schicht entwickelt (siehe Abschnitt Geröllanalysen und Tab. 1).

Der fossile B oden, der in der Kiesgrube Riedwanger das Liegende der Oberen Kiesmoräne bildet, wurde von SCHÄDEL \& WERNER (1963: 22) beschrieben und abgebildet. Er ist heute nicht mehr sichtbar, weil er im Zuge der Rekultivierung der Kiesgrube zugedeckt wurde. Als Ersatz dafür kann der in einer kleinen Kiesgrube E Ehrensberg neu aufgedeckte fossile Boden herangezogen werden (S. 145). Die rotbraune Farbe des sandigkiesigen Lehms, seine vollständige Entkalkung mit Tonanreicherung, seine Mächtigkeit von $1,7 \mathrm{~m}$, seine im Aufschluß horizontbeständige Lage sowie die verschiedene Geröllzusammensetzung der Kiese im Hangenden und Liegenden sind Merkmale echter fossiler Böden (HAAG 1979), wie sie bei Riedwanger und Ehrensberg entwickelt sind. (Weitere bodenkundliche Angaben S. 153).

Der kristallinreiche Schotter u n t e r dem fossilen Boden ist in der Kiesgrube Riedwanger in dem zum Talhang gelegenen Nagelfluhfelsen sowie in Nagelfluhfelsen am Hang unterhalb der Kiesgrube bei r 69020 h 23060 aufgeschlossen. Es handelt sich zumindest in den oberen Lagen ebenfalls um einen schlecht gerundeten, schlecht geschichteten Grobkies mit Blöcken und gekritzten Geschieben, also um einen moränennahen Schotter. Sein Kristallingehalt liegt mit 31 bis $44 \%$ deutlich über dem der oberen Kiesmoräne. Zu ihm wird auch der Kies mit 38 und $44 \%$ Kristallin in der Kiesgrube Fürstenwald (A 6) und der Geschiebemergel mit 42\% Kristallin wenig südlich davon gestellt.

Ein tiefer liegender, älterer fossiler Boden ist in Rottum im Wühlentobel und in der ehemaligen Kiesgrube $0,4 \mathrm{~km}$ südlich Rottum aufgeschlossen oder aufzugraben (bodenkundliche Angaben dazu S. 152).

Der Schotter im Liegenden des älteren fossilen Bodens unterscheidet sich stark von den 2 jüngeren, oben beschriebenen Kiesmoränen. Er enthält nur 2 bis $4 \%$ Kristallingerölle, aber $21 \%$ gelbe Kalke, und die Gerölle sind gut gerundet. Dies sind die Merkmale des Zeiler Schotters und des Heggbacher Schotters, die in Ubereinstimmung mit PENCK \& BRÜCKNER in die Günzeiszeit gestellt werden (SCHREINER \& EBEL 1981: 16; HAAG 1981: 91). Die Vorkommen bei Rottum gehören nach dem Schotterlängsprofil zu der Haisterkircher Rinne des Zeiler oder Heggbacher Schotters.

Hält man an dem fest, was PENCK im östlichen Rheingletscher als Günz gekennzeichnet hat - und das sollte man nach unserer Ansicht so weit wie möglich tun — dann sind die beiden kristallinreichen Kiesmoränen bei Rottum in die $\mathrm{Ri} \beta-\mathrm{und}$ in die Min d e le is z e it zu stellen. Für das rißeiszeitliche Alter der oberen Moräne sprechen außer der geschilderten Schichtenfolge auch die geringe Verwitterungstiefe und die hoch liegende Dolomitgrenze (Methode Fezer 1969: 10): Tabelle S. 142 oben.

Es ist noch anzumerken, daß sich der mindeleiszeitliche Schotter Rottum-abwärts mit steilerem Gefälle in die älteren Schotter eintieft. Im Bereich der Fürstenwaldkiesgrube (A 6) liegt die Untergrenze der Mindelschotter schon $5 \mathrm{~m}$ tiefer als der Günzschotter bei Hattenburg (A 5). Eine ähnliche Kreuzung von Schotterkörpern ist auch für die rißeis- 


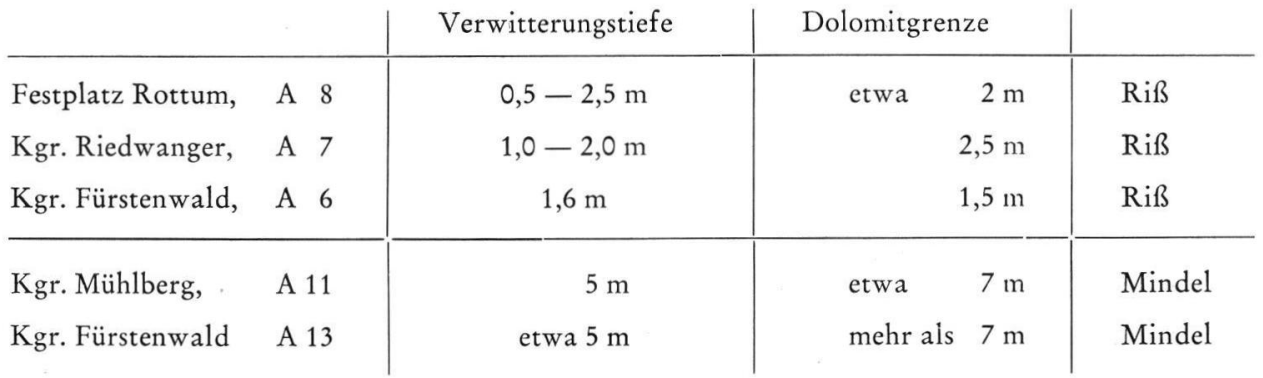

zeitlichen Schotter nachzuweisen, wie aus Aufschlüssen im Rottumtal N Ochsenhausen hervorgeht.

Weiterhin ist zu betonen, daß die 3-Gliederung bei Rottum Lücken aufweisen kann. So fehlen in ihr zum Beispiel die bei Füramoos und Unterpfauzenwald erkannte, zwischen Günz und Mindel eingeschobene Haslach-Eiszeit (SCHreINer \& Ebel 1981: 23). Sowohl nach der Geröllzusammensetzung als auch nach der Schotterverbindung von Rottum über Hürbel nach Laupheim (HAAG 1982: 250) besteht jedoch im vorliegenden Fall kein Zweifel, daß der untere kristallinreiche Schotter von Rottum zur Mindel-Eiszeit zu stellen ist. Die Ablagerungen der Haslacheiszeit sind im Rottumtal unterhalb von Füramoos offensichtlich ausgeräumt worden.

\section{Verwitterungstiefe auf Riß- und Mindelmoränen}

Die Möglichkeit, mit Hilfe von mehrfacher Überlagerung wie bei Rottum das Alter einer hangenden Moräne zu bestimmen, ist auf wenige Fälle beschränkt. Für die Darstellung der Verbreitung von Riß- und Mindelmoränen auf der geologischen Karte ist ein weiteres, im Gelände anwendbares Verfahren notwendig. Es lag nahe, dafür die allgemein bekannte, unterschiedliche Verwitterungstiefe heranzuziehen. Aus der Kenntnis, daß am Hang gelegene Kiesgruben auch in mindeleiszeitlichen Kiesen durch junge Hangabtragung sehr geringe Verwitterung von nur 0,5 bis $1 \mathrm{~m}$ zeigen können und daß sich andererseits in Mulden und am Hangfuß von Rißmoränen mehrere Meter entkalkte Verwitterungsmassen (z. T. Fließerden) anhäufen können, entwickelte sich der Versuch, die V e r w i t terungstiefe (= Entkalkungstiefe) auf der Gipfelfläche von flachen, allseitig abfallenden Kuppen zu erbohren (Handbohrgerät mit schwerem Hammer und Ziehgerät).

Auf solchen Kuppen ist die ursprüngliche Verwitterungsmächtigkeit noch am ehesten erhalten, denn es ist nichts oder nur wenig durch Bodenfließen weggegangen und es ist nichts zusätzlich abgelagert worden. Wie notwendig die Beschränkung auf allseitig abfallende Kuppen ist, zeigte sich in 3 Fällen, wo mangels solcher Kuppen an weniger geeigneten Stellen gebohrt und ein unbrauchbares Ergebnis erzielt wurde (Bohrg. 12, 18 und 21).

Im Gebiet Warthausen-Ringschnait-Ochsenhausen-Rottum-Ellwangen wurden auf der Karte geeignete Kuppen ausgesucht und auf ihnen in den Jahren 1979-1981 33 Bohrungen ausgeführt - ein gewiß etwas primitives, dafür anstrengendes, aber insgesamt erfolgreiches Verfahren.

Als rißeiszeitlich wurden Moränen eingestuft, deren Verwitterungstiefe geringer als $3 \mathrm{~m}$ ist. Das Minimum von 0,5 $\mathrm{m}$ wurde in der Bohrung 3 auf einer Kuppe des Riß-Doppelwalls gefunden. Die als mindele is z e it lich eingestuften Moränen weisen Verwitterungstiefen von $4 \mathrm{~m}$ und mehr auf. 
In den Bohrungen 2 (Windberg) und 7 (Gurgel) mit nur 3,8 und 3,9 m Verwitterungstiefe wird aufgrund der Nachbarschaft von Bohrungen mit größerer Verwitterungstiefe bei vergleichbarer geologischer Lage auf mindelzeitliche Moränen geschlossen. Ansonsten überwiegen auf Mindelmoränen Verwitterungstiefen um $5 \mathrm{~m}$ und reichen bis $10 \mathrm{~m}$. In vielen Fällen wurde die Bohrung bei 4,8 $\mathrm{m}$ abgebrochen.

Die großen Unterschiede in der Verwitterungstiefe auf Mindelmoränen sind auch in Tagesaufschlüssen zu beobachten. Sie hängen mit der wechselhaften Wasserzügigkeit in Abhängigkeit vom Kleinrelief, von den Deckschichten, von der Durchlässigkeit und wohl noch anderen Faktoren zusammen. Als Beispiel dafür sei der Aufschluß Mühlberg, $4 \mathrm{~km}$ SW Rot, abgebildet (Abb. 3).

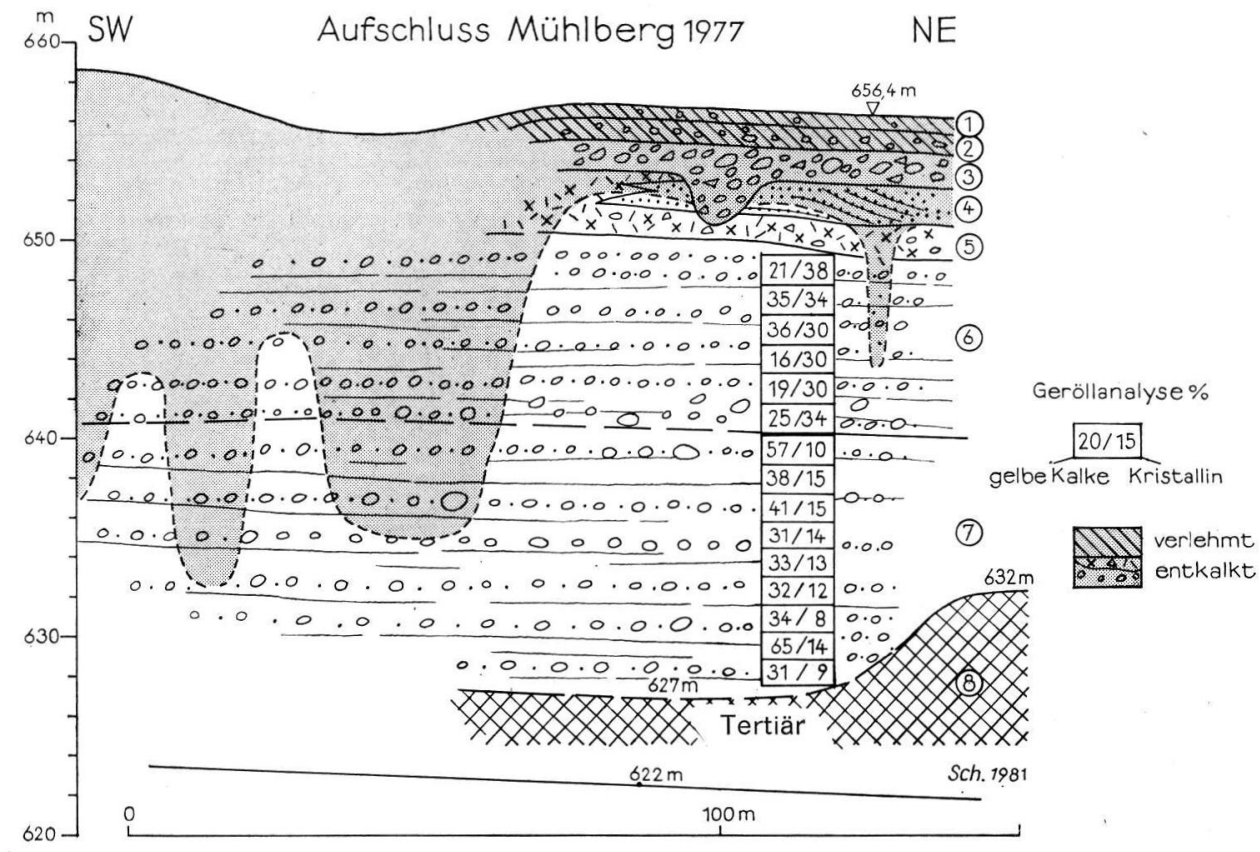

Abb. 3: Aufschluß Mühlberg 1977 (TK 25 Wurzach, r 74000 h 77 200)

Kiesentnahme für den Bau des Staudamms im Pfaffenrieder Tal.

Geröllanalysen HaAg; geol. Aufnahme Ebel, HaAg u. Schreiner.

1. $0,8 \mathrm{~m} \mathrm{~L} \mathrm{e} \mathrm{h} \mathrm{m,} \mathrm{gelb,} \mathrm{marmoriert,} \mathrm{kryoturbat} \mathrm{verlagerte} \mathrm{Gerölle} \mathrm{(Decklehm)}$

2. $1 \mathrm{~m} \mathrm{~L} \mathrm{e} \mathrm{h} \mathrm{m,} \mathrm{braun,} \mathrm{kiesig-sandig} \mathrm{(Verwitterungslehm)}$

3. $1,8 \mathrm{~m}$ B lö ck e mit Kies, braun, lehmig, Blöcke bis $40 \mathrm{~cm}, \mathrm{z}$. T. Dachziegelschichtung, viel Amphibolite (fluviale Blocklage, Mindel Rückzug)

4. $2,5 \mathrm{~m} \mathrm{~S}$ a nd, mit Kieslagen, graubraun, z. T. schräggeschichtet, im W mit Geschiebemergel verzahnt (glazifluvialer Sand, Mindel)

5. 1-3 m G e s ch i e be me r ge 1, grau, z. T. Nagelfluh, gekritzte Geschiebe (Mindel-Grundmoräne)

6. $8 \mathrm{~m} \mathrm{Ki}$ es-S a nd, blaugrau, geschichtet, Gerölle bis $20 \mathrm{~cm}$, Rundung z. T. gut, z. T. schlecht, Sandlagen, hoher Kristallingehalt (oberer Mühlberger Schotter, Mindelglazifluvial)

7. $14 \mathrm{~m} \mathrm{~K}$ ie s-S a nd, graugelb, geschichtet, Gerölle bis $30 \mathrm{~cm}$, meist gut gerollt, mittlerer Kristallingehalt (unterer Mühlberger, Haslach-glazifluvial in Rinnenlage, ScHREINER \& EBEL 1981, Abb. 6)

8. M e r g e 1, graugelb, feinsandig (Obere Süßwassermolasse) 


\section{Das Zungenriß (Rz)}

Das Ergebnis der Untersuchung der Verwitterungstiefe, verknüpft mit den Befunden an Aufschlüssen (z. B. Rottum) ist in Abb. 1 dargestellt: Vor der Riß-Doppelwall-Endmoräne schieben sich im Bereich von Tälern zungenförmig ausgebreitete Decken von Moränen mit geringer Verwitterungstiefe in das Gebiet der tief verwitterten Mindelmoränen. Die Moränen mit geringer Verwitterungstiefe werden als älterer Teil der Rißeiszeit betrachtet und als $\mathrm{Zung}$ en ri $ß$ bezeichnet. Zwischen und neben den Zungen, deren Abgrenzung nur in Einzelfällen scharf zu ziehen ist, liegen meist 10 bis $30 \mathrm{~m}$ höhere Hügel und Höhenzüge aus Mindelmoräne wie z. B. der Aspen NW Ringschnait oder der Höhenzug Bubenloch-Geschlötter zwischen der Dürnach- und der Rottumzunge. Daraus wird geschlossen, daß die Gletscher des Zungenriß von geringer Mächtigkeit und wahrscheinlich warmtemperiert waren, im Gegensatz zu dem mit fast gerader Front vorstoßenden Gletscher der Riß-Doppelwalls.
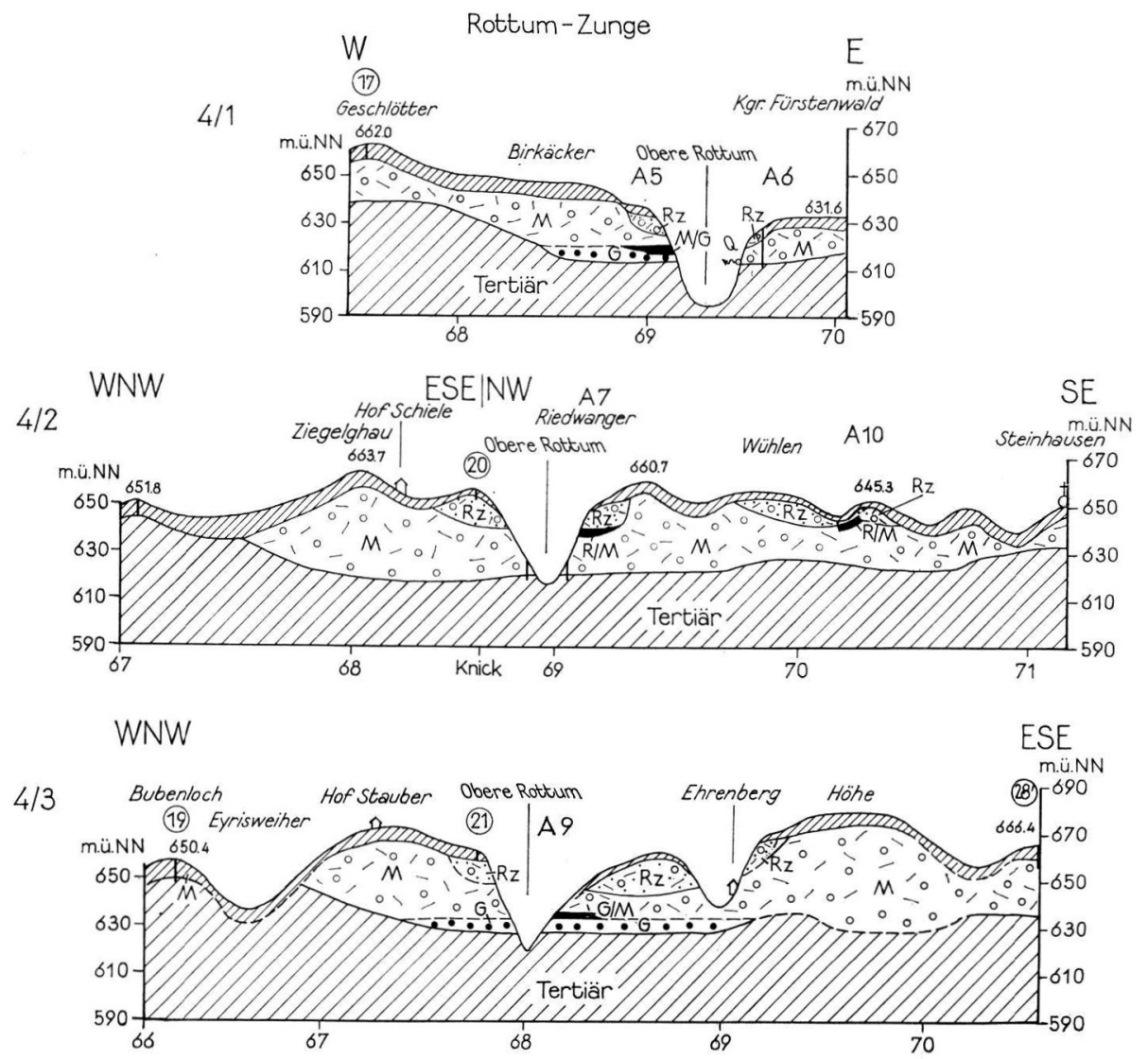

Abb. 4: Quartärgeologischer Querschnitt durch die Rottum-Zunge, 12,5 x überhöht.

A 5 - A 10 Aufschluß-Nr.

Rd Riß-Doppelwall-Endmoräne

$\mathrm{Rz} \quad$ Zungen-Riß, Moränen und Schotter

$\mathrm{R} / \mathrm{M} \quad$ Fossiler Boden Riß/Mindel

M Mindel, Moränen und Schotter

M/G fossiler Boden Mindel/Günz

G Günz, Heggbacher Schotter 
Gegenüber dem Tertiärhochgebiet bei Bubenloch stellt die Umgebung des Rottumtals eine alte Tiefenlinie dar, in der an der Sohle die günzzeitlichen Heggbacher Schotter, darauf die Mindelschotter und schließlich die Moränen und Schotter des Zungenriß abgelagert wurden.

\subsection{Rottumzunge (Abb. 4)}

Von Bellamont und Bebenhaus, wo kiesige Stauchmoränen mit nur 1 bis $2 \mathrm{~m}$ Verwitterungstiefe und einer Dolomitgrenze von 2 bis $3 \mathrm{~m}$ aufgeschlossen sind, entwickelt sich eine rißeiszeitliche Moränenzunge Rottum-abwärts bis Hattenburg und Fürstenwaldkiesgrube (A 6, Abb. 2 und 4). Weithin sind die Rißmoränen von Fließerde und Decklehm verhüllt (z. B. bei Bohrg. 21 und Kammerlander).

Eine Nebenzunge nach NE wird bei Steinhausen/Rottum angenommen, denn in der Kiesgrube 0,8 km NE des Dorfes ist folgende Schichtfolge zu sehen (A 10, R 70200 H 21 860):

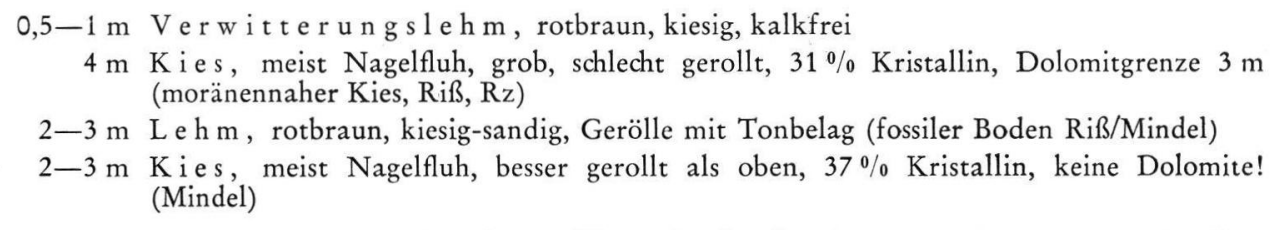

Das rißeiszeitliche Alter des oberen Kieses ist durch seine Lage über dem fossilen Boden, der dem in der Kiesgrube Riedwanger (S. 140) entspricht, durch die hochliegende Dolomitgrenze und die geringe Verwitterungstiefe begründet.

Die Höhen W Steinhausen erwiesen sich in den Bohrg. 28 und 28' mit 3,6 und 3,5 m Verwitterungstiefe als Mindelmoräne, wobei einige dm Bodenerosion auf dem Ackerland anzunehmen sind. Im Gegensatz dazu ist SW Steinhausen bei den Bohrg. 29 und 30 mit nur 1,5 und 2,4 m Verwitterungstiefe wieder eine Zunge des älteren Riß anzunehmen. Sie wird durch Mindelmoräne bei Bohrg. 31 mit mehr als 4,8 m Verwitterung begrenzt.

Westlich von Rottum liegt das Zungenriß vom Gewann Höhe über Bohrg. 20 bis Enketsweil bei Hattenburg auf einer um 5 bis $10 \mathrm{~m}$ tieferen Geländestufe als die westlich anschließenden Höhen mit größerer Verwitterungstiefe (Bohr. 11 und 9, Abb. 4).

Südlich von der Rottumzunge sind bis jetzt außerhalb des Riß-Doppelwalls keine Moränen des Zungenriß festgestellt worden. In den Bohrungen 23 bis 27 sind tief verwitterte Mindelmoränen angetroffen worden. Demnach wird südlich von Bellamont die äußerste Rißmoräne von der Riß-Doppelwall-Endmoräne, wie sie bei SCHREINER \& EBEL (1981, Taf. 1) dargestellt wurde, gebildet.

\subsection{Dürnachzunge (Abb. 5)}

Westlich des Tertiärhochgebietes Bubenloch-Geschlötter (E Mittelbuch), das von tief verwitterten Mindelmoränen bedeckt ist (Bohrg. 15, 17 u. 19), zieht sich beiderseits der Dürnach eine Zunge mit Moränen geringerer Verwitterungstiefe nach $\mathrm{N}$. Sie ist durch die Moränenhügel um Ringschnait (P. 644,7, 1,5 m Verwitterung), die hier großenteils mit Flugsand bedeckt sind (Bohrg. 14) und durch die Kiesgrube 1,5 km NNE Ringschnait belegt (A 4, r 66250 h 28 000). 
$5 / 1$

NW

Dürnach-Zunge

SE

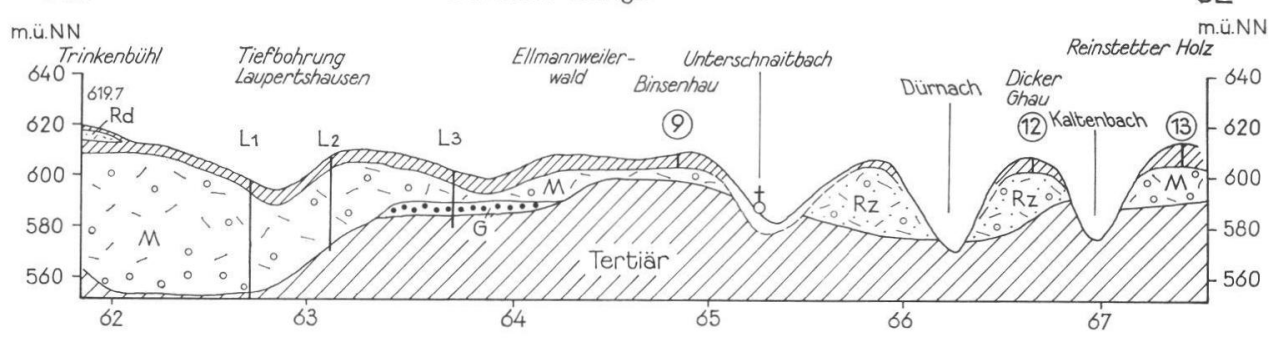

$5 / 2$

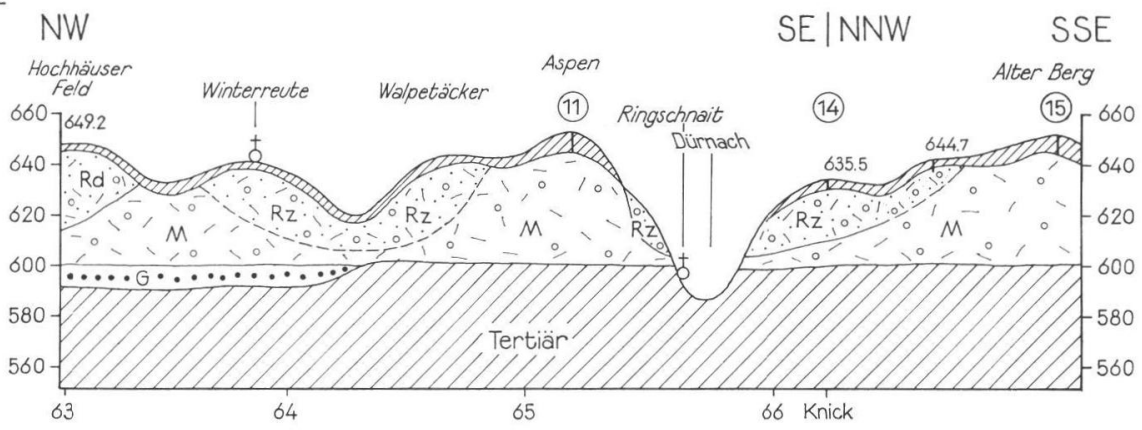

$5 / 3$

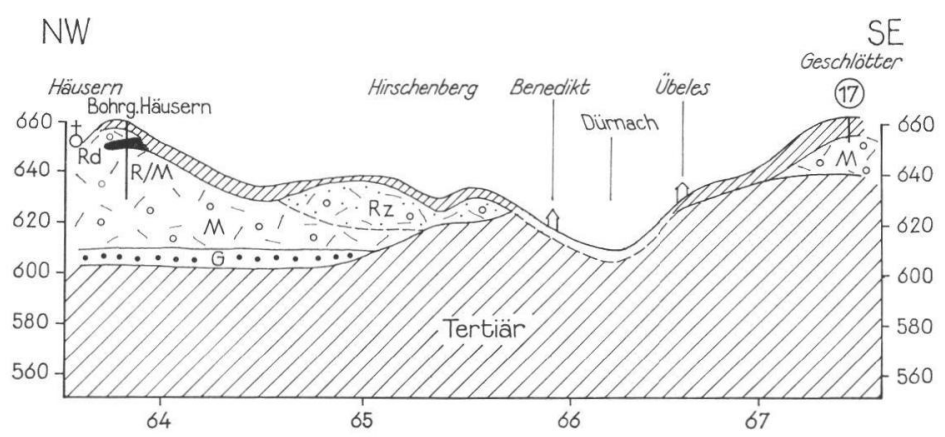

Abb. 5: Quartärgeologischer Querschnitt durch die Dürnach-Zunge.

Zeichenerklärung s. Abb. 4. 12,5 x überhöht.

Sie hat folgenden Aufbau:

- Ebene Oberfläche -

1. 1,5- $3 \mathrm{~m} \mathrm{~L} \mathrm{e} \mathrm{h} \mathrm{m,} \mathrm{braun,} \mathrm{kiesig,} \mathrm{kalkfrei} \mathrm{(Verwitterungslehm)}$

2. $1 \mathrm{~m} \mathrm{Geschiebemergel,z.} \mathrm{T.} \mathrm{Nagelfluh,} \mathrm{graugelb,} \mathrm{mit} \mathrm{gekritzten} \mathrm{Geschieben,}$ kalkig, $18 \%$ Kristallin, $2 \%$ gelbe Kalke (Rißmoräne, $\mathrm{Rz}$ )

3. $0-2,5 \mathrm{~m} \mathrm{Lehm}$, braun, kiesig, unvollständig entkalkt, Dolomitaschen und kalkige Kerne von Geröllen, mit Durchgriffen in 2. (Pseudoboden durch Durchgriffe von oben)

4. $6-8 \mathrm{~m} \mathrm{~K} \mathrm{i} \mathrm{e} \mathrm{s,} \mathrm{grob,} \mathrm{Blöcke} \mathrm{bis} 20 \mathrm{~cm}$, geschichtet, $25 \%$ Kristallin, $4 \%$ gelbe Kalke, Dolomitgrenze etwa $3 \mathrm{~m}$ unter 1 . (Riß, moränennaher Kies)

5. $\quad-1,5 \mathrm{~m} \mathrm{Geschiebemergel,} \mathrm{nach} \mathrm{S}$ noch mächtiger werdend, nach $\mathrm{N}$ in Blocklagen übergehend, gekritzte Geschiebe

6. $8-10 \mathrm{~m} \mathrm{~K} \mathrm{i} \mathrm{e} \mathrm{s,} \mathrm{meist} \mathrm{unter} \mathrm{Schutt} \mathrm{(nach} \mathrm{Längsschnitt} \mathrm{wahrscheinlich} \mathrm{Mindel)}$

Kiesuntergrenze (erbohrt) $582 \mathrm{~m}$. 
Morphologisch deutlich ist die in die ältere und höhere Umgebung eingelagerte Rißmoräne 0,5 bis $1,5 \mathrm{~km} \mathrm{~N}$ Ringschnait. Nach unserer Auffassung ist die Rißzunge nach $\mathrm{N}$ bis ungefähr $1 \mathrm{~km} \mathrm{SW}$ Wennedach vorgestoßen und hat von hier aus das rißeiszeitliche Schotterfeld von Maselheim, das mit der höheren Rißterrasse von Baltringen zu verbinden ist, geschüttet.

Südlich um den Aspen herum, der eine tief verwitterte Moränendecke trägt (Bohrg. 11: 4,5 m), dringt die Rißmoräne über das Neubaugebiet W Ringschnait mit einer kleineren Zunge in das Schnaitbacher Tal NE von Winterreute vor (Bohrg. 10 und benachbarte Kiesgruben mit Verwitterungstiefen unter $3 \mathrm{~m}$ ). Unter den zungen- und rinnenartig eingelagerten Rißmoränen und -schottern liegen mindelzeitliche Moränen. Die daraus hervorgehenden Schotter sind bei abnehmendem Kristallingehalt über Ringschnait nach W zu verfolgen, wo sie mit dem Hauptschotterstrom der Mindeleiszeit (Tannheim-Laupheimer Schotter, HAAG 1982) zu verbinden sind. Der älteste hier auftretende Schotter gehört zu dem vom Hochgelände über Häusern-Ellmannsweiler herziehenden günzzeitlichen Heggbacher Schotter.

Im SW bei Winterreute erheben sich die Höhen des Rißendmoränen-Doppelwalls über die älteren Flächen. In der Bohrung Häusern wurde ein fossiler Boden zwischen Riß (Rd) und Mindel angetroffen (HAAG 1982: 257):

- $2 \mathrm{~m}$ Lehm, braun, kantige Gerölle, z. T. kalkig

- $5 \mathrm{~m}$ im Aufschluß neben der Bohrung: Kies u. Moräne, kalkig (Zungen-Riß 15-25\% Krist.)

- $10 \mathrm{~m}$ Lehm, braun, kalkfrei, z. T. von oben aufgekalkt (fossiler Boden Riß/Mindel)

- $29 \mathrm{~m}$ Kies u. Schluff, grau, unten gelb, gekritzte Geschiebe, kalkig (Mindel, $30 \%$ Kristallin)

\subsection{Rißtal-Zunge (Abb. 6)}

Im Rißtal ist das Zungenriß wahrscheinlich bis nach Eichelsteig (3,5 km N Warthausen) vorgestoßen, denn in der dortigen Kiesgrube beobachtete WERNER (1978: 92) unter dem Alberweiler Rißschotter, der mit dem Doppelwallriß zu verknüpfen ist, einen Geschiebemergel, der aufgrund seiner tiefen Lage (etwa $530 \mathrm{~m}$ ) nicht mindelzeitlich sein kann.

Zum Zungenriß wird auch der Aufschluß, der beim Bau der Umgehungsstraße Biberach, 0,5 km S Barabein, entstanden ist, gestellt (A 1, R 61650 H 34 050):

Oberfläche $550 \mathrm{~m}$, nach W fallender Hang

$1,1 \mathrm{~m} \mathrm{~L} \mathrm{e} \mathrm{h} \mathrm{m,} \mathrm{rotbraun,} \mathrm{kiesig} \mathrm{(Verwitterungslehm)}$

$1,8 \mathrm{~m} \mathrm{Geschie} \mathrm{be} \mathrm{merge} 1$, hellgrau, gekritzte Geschiebe, mit kiesiger Zwischenlage

$3 \mathrm{~m}$ Kies, z. T. Nagelfluh, Blöcke bis $20 \mathrm{~cm}, 17 \%$ Kristallin, $6 \%$ gelbe Kalke, Dolomitgrenze $1-2 \mathrm{~m}$ unter Schicht 1

$4 \mathrm{~m} \mathrm{Kies}$, geschichtet, $22 \%$ Kristallin, $7 \%$ gelbe Kalke, oben rote Fe-Oxyd-Ausscheidungen Am Hang geht der Kies noch $10 \mathrm{~m}$ tiefer.

Von hier aus nach $\mathrm{S}$ war Geschiebemergel mit geringer Verwitterungstiefe (1 bis $3 \mathrm{~m}$ ) den Straßenbaustellen entlang bis vor Mettenberg zu verfolgen. Dort lag in einer Brückenbaugrube (A 3, r 62050 h 30680 ) 4 m tief verwitterter Silt und Feinsand mit wenigen Geröllen, die als hochgelegene mindelzeitliche Beckensedimente zu deuten sind. Darüber folgt 2,2 m moränennaher Kies mit Blöcken bis $40 \mathrm{~cm}$, der nur 1,2 m tief verwittert ist und von der nahe gelegenen Rißdoppelwall-Endmoräne stammt. Zwischen der Zunge von Winterreute-Schnaitbach und der Rißtalzunge nördlich Mettenberg ist demnach das hoch gelegene Gebiet von Boschach bis Königshofen (Bohr. 7) nicht vom Zungenriß überdeckt worden. 

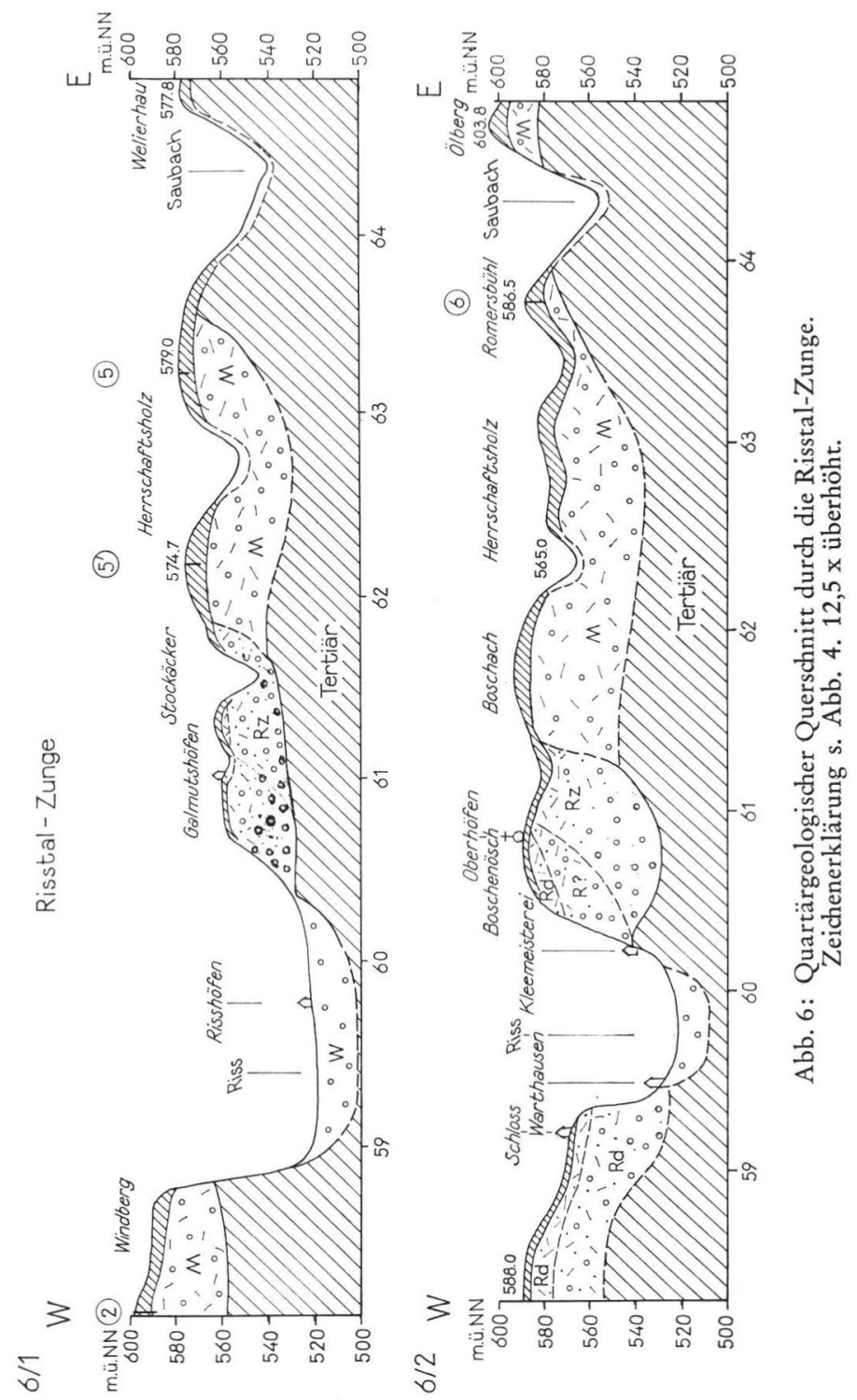

Der braune Lehm zwischen frischem Kies, der in der alten Kiesgrube E Galmutshöfen zu sehen war (r61540 h 33 600, Graul 1950; EICHLER 1970: 100) erwies sich nach einer Grabung nicht als fossiler Boden, sondern als abgerutschter Oberflächenlehm, der durch nachrollenden Kies bedeckt worden war. Wie der benachbarte Kies bei Barabein (A 1, siehe oben) wird deshalb auch der Kies von Galmutshöfen insgesamt in die Rißeiszeit gestellt. Er ist von der Moräne des Rz bedeckt.

Westlich des Rißtales sind am Windberg in hoher Lage $(591 \mathrm{~m})$ mit den Bohrungen 1 und 2 wieder Mindelmoränen und südlich davon der äußere Wall der Riß-Doppelwall- 


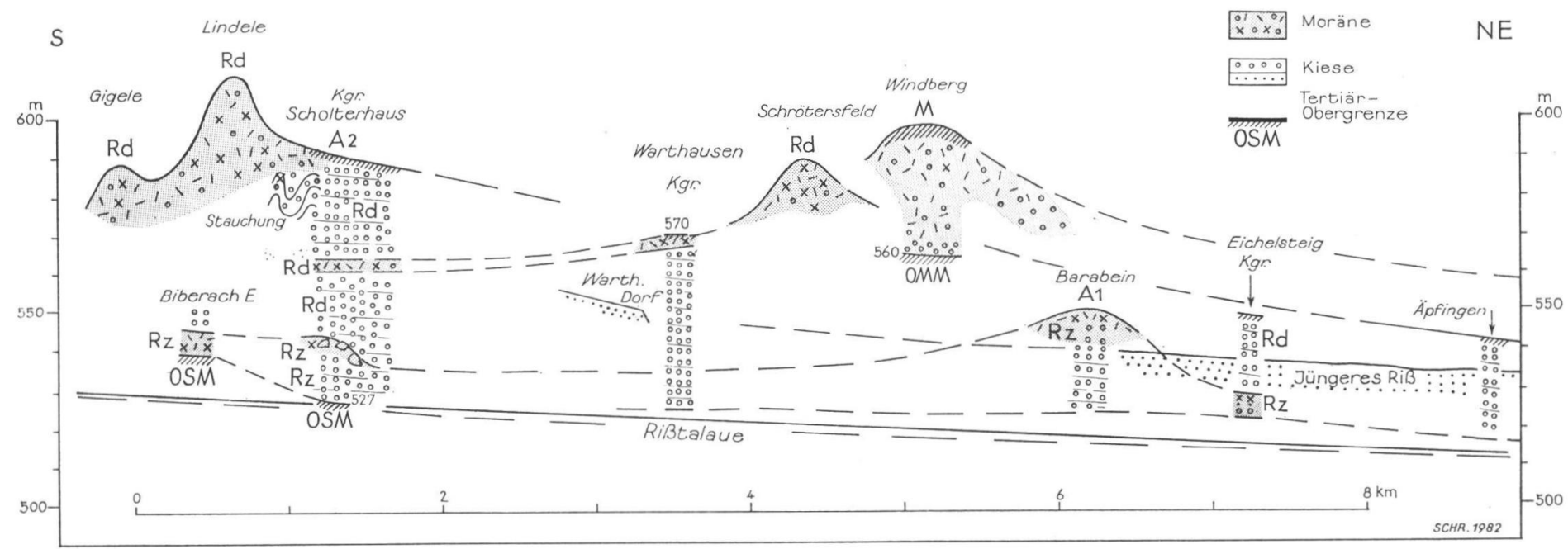

Abb. 7: Längsschnitt durch das Rißtal (vgl. Text S. 150)

$\mathrm{Rz}$ Zungenriß (= älteres Riß) $\begin{array}{ll}\text { Mindel } & \text { Rd Doppelwallriß (= mittleres Riß) }\end{array}$

OSM Obere Süßwassermolasse OMM Obere Meeresmolasse.

Unter Verwendung von Angaben aus Penck 1883, Krauss 1930, Weidenbach 1937, Graul 1968 u. Mader 1976. 
Endmoräne (Bohrg. 3 und 4) erkannt worden. Das Zungenriß dürfte demnach im Bereich des heutigen Rißtales östlich vom Windberg nach $\mathrm{N}$ bis Eichelsteig vorgestoßen sein. Weiter im W stehen neue Untersuchungen zur Gliederung im Zungenriß und Doppelwallriß noch aus.

\section{Ablauf der Ereignisse im Rißtal und Vergleiche (Abb. 7)}

Die tiefe Lage des Zungenriß bei Eichelsteig und Barabein stimmt mit der unteren Moräne oder deren Reste in der bekannten Kiesgrube am Scholterhaus überein (A 2 r 58900 h 30 450, 10 m über Sohle, Mader 1976: 61; Schreiner 1980: 23). Daraus ist zu schließen, daß das Rißtal zu Beginn des ersten Rißvorstoßes (= Zungenriß) ungefähr bis zur Höhe der heutigen Talaue ausgeräumt war. Nach der bei Barabein etwa $30 \mathrm{~m}$ mächtigen Verfüllung mit Kies und Moräne durch das Zungenriß erfolgte eine Erosionsphase mit teilweiser Ausräumung des Zungenriß, was einen beträchtlichen Eisrückzug voraussetzt. Danach folgte die am Scholterhaus $40 \mathrm{~m}$ mächtige Aufschotterung vor dem Rißdoppelwallvorstoß mit der mittleren Moräne (Geschiebemergelband $35 \mathrm{~m}$ über der Sohle in der Kgr. Scholterhaus), die mit dem äußeren Doppelwall bei Warthausen-Schrötersfeld (Bohrg. 4) zu verbinden ist. Die oberen Kieslagen in der Kgr. Scholterhaus mit der Stauchmoräne im südlichsten, ältesten Teil der Kiesgrube gehören zum inneren Wall der Riß-DoppelwallEndmoräne (= Lindele-Endmoräne).

Im Rißtal ist somit ein ähnlicher Ablauf der rißzeitlichen Ereignisse festzustellen wie bei Meßkirch (Werner 1975: Beil. 8) und Saulgau (Schreiner 1979: 25). Das „ältere Riß“ entspricht dem Zungenriß (Rz), das „mittlere Riß“ dem Doppelwall (Rd) und dazwischen dürfte das Paulter Interstadial (Schädel 1955; Schreiner 1979: 22) liegen. Das „jüngere Riß“ mit der Laupheimer Rißterrasse folgte nach dem Doppelwall-Riß und wird hier nicht behandelt.

\begin{tabular}{|c|c|}
\hline WEIDENBACH 1937a & hier vorgelegte Gliederung \\
\hline \multirow{2}{*}{$\begin{array}{l}\text { Laupheimer Rißterrasse } \\
\text { R II }\end{array}$} & jüngeres Riß \\
\hline & $\begin{array}{l}\text { Rückzug } \\
\text { Erosion }\end{array}$ \\
\hline R II Doppelwall & mittleres Riß = Doppelwall-Riß $(\mathrm{Rd})$ \\
\hline \multirow[t]{2}{*}{$\begin{array}{l}\text { R I Vorstoßschotter } \\
\text { und Moränen }\end{array}$} & $\begin{array}{l}\text { Rückzug } \\
\text { Erosion }\end{array}$ \\
\hline & älteres Riß $=$ Zungenriß $(\mathrm{Rz})$ \\
\hline
\end{tabular}

\section{Geröllanalysen}

Die im vorangehenden Text schon mehrfach erwähnte Geröllzusammensetzung in Kiesen und Moränen wurde durch Geröllanalysen, wie sie in großer Zahl von HAAG (1979, 1981, 1982) und Schreiner \& Ebel (1981) durchgeführt wurden, ermittelt. Es hat 
sich gezeigt, daß die Geröllanalyse zusammen mit der Schichtlagerung ein brauchbares Hilfsmittel zur Verbindung gleichartiger und zur Trennung verschiedenartiger Kiese ist. Es gibt jdoch keine durchgehende Standardzusammensetzung für Riß oder Mindel. Man muß vielmehr für jede Abflußrinne die ihr eigene Zusammensetzung finden. Von großem Einfluß sind Veränderungen der Geröllzusammensetzung durch Einarbeitung älterer Kiese.

Der für die Unterscheidung wichtigste Bestandteil sind Gerölle aus Gneisen, Glimmerschiefer, Graniten und Amphiboliten - zusammen kurz als Kristallin (Kr) bezeichnet.

Tabelle 1: Geröllanalysen ( $\%$ der Geröllsumme)

$$
\phi 2-6 \mathrm{~cm}
$$

\begin{tabular}{lcccccccc|cc|cc|c}
\hline Kiesgrube & & Nr. & $\mathrm{S}$ & $\mathrm{Kf}$ & $\mathrm{Kb}$ & $\mathrm{kS}$ & $\mathrm{D}$ & $\mathrm{kG}$ & $\mathrm{Q}$ & $\mathrm{Kr}$ & $\mathrm{A}$ & $\mathrm{Geol}$ \\
\hline Hattenburg & $\mathrm{A}$ & 5 & 1 & 155 & 46 & 3 & 0 & 21 & 6 & 1 & $\mathbf{2 3}$ & 5 & $\mathrm{Rz}$ \\
& & 2 & 283 & 17 & 16 & 0 & 16 & 11 & 4 & $\mathbf{3 6}$ & 12 & $\mathrm{M}$ \\
\hline Fürstenwald & $\mathrm{A}$ & 6 & 3 & 263 & 35 & 11 & 5 & 12 & 15 & 2 & $\mathbf{2 0}$ & 5 & $\mathrm{Rz}$ \\
& & 4 & 272 & 36 & 14 & 0 & 6 & 5 & 2 & $\mathbf{3 8}$ & 19 & $\mathrm{M}$ \\
\hline Riedwanger & A & 7 & 5 & 420 & 49 & 11 & 0 & 6 & 7 & 1 & $\mathbf{2 5}$ & 6 & $\mathrm{Rz}$ \\
& & 6 & 268 & 40 & 17 & 0 & 2 & 4 & 2 & $\mathbf{3 5}$ & 14 & $\mathrm{M}$ \\
\hline Rottum- & & 7 & & 66 & 5 & 9 & 1 & 5 & 2 & $\mathbf{1 2}$ & 3 & $\mathrm{Rz}$ \\
Festplatz & A & 8 & 8 & 241 & 25 & 22 & 0 & 13 & 5 & 1 & $\mathbf{3 2}$ & 13 & $\mathrm{M}$ \\
\hline Rottum- & & 9 & 234 & 35 & 10 & 0 & 7 & 11 & 0 & $\mathbf{3 5}$ & 13 & $\mathrm{M}$ \\
Wühlentobel & A & 9 & 10 & 425 & 42 & 41 & 4 & 0 & 11 & 0 & $\mathbf{2}$ & 0 & $\mathrm{G}$ \\
\hline Ehrensberg & A 10 & 11 & 213 & 40 & 8 & 7 & 1 & 10 & 2 & $\mathbf{3 1}$ & 2 & $\mathrm{Rz}$ \\
& & 12 & 234 & 39 & 9 & 6 & 0 & 7 & 2 & $\mathbf{3 7}$ & 10 & $\mathrm{M}$ \\
& & 13 & 180 & 44 & 12 & 9 & 0 & 9 & 3 & $\mathbf{2 3}$ & 7 & $\mathrm{M}$ \\
\hline Ringschnait & A & 4 & 14 & 268 & 51 & 4 & 5 & 0 & 6 & 1 & $\mathbf{2 5}$ & 11 & $\mathrm{Rz}$ \\
& & 15 & 251 & 60 & 5 & 5 & 9 & 8 & 1 & $\mathbf{1 6}$ & 5 & $\mathrm{M}$ ? \\
\hline Barabein & A & 1 & 16 & 222 & 65 & 6 & 5 & 4 & 3 & 0 & $\mathbf{1 7}$ & 6 & $\mathrm{Rz}$ \\
\hline Fürstenwald & A 13 & 17 & 170 & 34 & 23 & 0 & 0 & 8 & 2 & $\mathbf{3 3}$ & 15 & $\mathrm{M}$ \\
\hline Häusern & A 12 & 18 & 205 & 71 & 2 & 0 & 8 & 4 & 1 & $\mathbf{1 4}$ & 2 & $\mathrm{Rd}$ \\
& & 19 & 298 & 51 & 11 & 0 & 4 & 6 & 1 & $\mathbf{2 7}$ & 6 & $\mathrm{Rd}$ \\
\hline
\end{tabular}

A 1 bis A 13 siehe Abb. 1 und Aufschlußverzeichnis S. 160

S Geröllsumme

Kf Kalke, frisch

$\mathrm{Kb}$ Kalke, braun u. gelb

kS kalkhaltige Sandsteine

D Dolomite

G Günz

M Mindel
kG kieselige Gerölle (Hornsteine u. Quarzite)

Q Quarz

$\mathrm{Kr}$ Gesamtkristallin

A Amphibolit (in $\mathrm{Kr}$ enthalten)

$\mathrm{Rz}$ Zungenriß

Rd Doppelwall-Riß 
In Tabelle 1 sind von Nr. 1 bis 8 Zweiergruppen von Analysen aus Kiesgruben aufgeführt, wobei die obere Reihe jeweils rißzeitlichen, die untere mindelzeitlichen Kiesen entstammt. Dasselbe gilt für 11 und 12. Bei Riedwanger und Ehrensberg ist diese Trennung durch fossile Böden belegt. Die Kiese von $\mathrm{Rz}$ liegen im Kristallingehalt durchweg niedriger als Mindel-Kiese (M), die zwischen 31 und $38 \% \mathrm{Kr}$ aufweisen. Dabei ist der Amphibolitanteil bei Mindel in der Regel deutlich höher als bei Rz. Dasselbe gilt in der Regel für braune Kalke (Kb).

Eine Abweichung stellt Nr. 13 dar, die ihrer Lage nach (4 m unter 12 liegend)nur mindelzeitlich sein kann. Ihr zu niedriger Kristallingehalt ist durch Aufnahme kristallinarmer Schotter zu erklären, wie sie im südwestlichen Herkunftsgebiet bei Füramoos liegen (Schreiner \& Ebel 1981: 39) und hier in Tabelle 1 unter Nr. 10 mit nur 2\% Kristallin erscheinen (Heggbacher Schotter, günzzeitlich).

Vielleicht ist das fragliche Mindel von Ringschnait (Nr. 15) mit nur $16 \%$ Kristallin ähnlich zu erklären.

Nr. 16 und 17 sind Einzelanalysen weit voneinander entfernter Vorkommen, wobei das Mindelalter von 17 durch $5 \mathrm{~m}$ tiefe Verwitterung und das Rißalter von 16 durch geringe Verwitterung (S. 147) gestützt wird.

Nr. 18 und 19 sind Beispiele für das Doppelwall-Riß (Rd), dessen Kristallingehalt noch tiefer liegen kann als bei Rz. Dann ist der Anteil an frischen, blaugrauen helvetischen Kalken besonders hoch.

\section{Untersuchungen an fossilen Böden}

Auf die Problematik der fossilen Böden oder deren Reste wurde bereits früher hingewiesen (HaAG 1979). Die dort geforderten Mindestbedingungen für die Anerkennung eines fossilen Bodens wurden auch bei dieser Untersuchung angewandt. Darüber hinaus kamen die mikromorphologischen Untersuchungsmethoden verstärkt zur Anwendung. Von den vielen schon von WEIDENBACH (1940) beschriebenen fossilen Böden sei jenes noch heute gut zugängliche Profil in Rottum hinter dem Hof im W üh le n to bel (A 9) beschrieben. Von oben nach unten ist im Aufschluß zu sehen:

1. $-1,5 \mathrm{~m}$ K i e s, grob, kalkhaltig, Gerölle oft nur kantengerundet, sehr kristallinreich (s. Tab. 1, Probe Nr. 9)

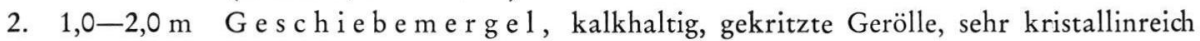

3. $0,3-0,5 \mathrm{~m}$ kiesiger Lehm, Gerölle zerbrochen und die Bruchstücke verlagert, kalkfrei, kristallinarm

4. 0,2-0,3 m kiesige r L e h m, Gerölle nur leicht deformiert und die Bruchstücke nicht verlagert, kalkfrei, kristallinarm

5. $0,3-0,5 \mathrm{~m}$ s a n dig-1 e h mige r $\mathrm{K}$ i e s, Gerölle intakt, in den oberen Bereichen kalkfrei, nach unten einzelne Nester von kalkhaltigem grauen Kies, kristallinarm

6. $0,5-1,5 \mathrm{~m}$ s a ndige r Kies, gut gerundet, kalkhaltig, kristallinarm s. Tab. 1, Probe Nr. 10).

Der geringe Kristallingehalt des Verwitterungslehmes der Horizonte 3, 4 und 5 weist darauf hin, daß die Verwitterungszone aus dem liegenden kristallinarmen Kies entstanden ist.

Die Präparation der Proben zur Herstellung von Bodendünnschliffen wurde nach Altemüller (1962) durchgeführt.

Die mikromorphologischen Untersuchungen des fossilen Bodens von Rottum-Wühlentobel (A 9) zeigen innerhalb des Horizontes 3 schlecht sortierten Ton mit Schluffbeimengung ohne Schichtungsmerkmale. Risse innerhalb der Körner sowie der aus schlecht sortiertem Ton bestehenden Aggregate sind mit einer geringmächtigen Tontapete mit 
orientierter Doppelbrechung (im polarisierten Licht) ausgekleidet. Im darunter folgenden Horizont 4 sind die gut geschichteten (im polarisierten Licht Doppelbrechung zeigende) Tonbeläge zerrissen und schwimmen in einer Masse aus schlecht sortiertem Ton (Taf. 1, Fig. 1). Altemüller (1960: 71) nennt dies ein brecciös es G ef üge. Entsprechende Strukturen in der Tonsubstanz beschrieben SCHÄDEL \& WERNER (1963: 18) aus dem fossilen Boden von Neufra bei Riedlingen. Innerhalb von Körnern mit Lösungshohlräumen, so z. B. innerhalb eines Glaukonitsandsteines, sind die geschichteten gut sortierten Tonablagerungen unversehrt erhalten (Taf. 1, Fig. 2). Glimmer, insbesondere Biotite, sind an den Rändern aufgeweitet oder gar in einzelne Blättchen aufgelöst (im Schliffbild der Taf. 1, Fig. 3 als Leisten erscheinend). Die feinen Schichtungsstrukturen der Toneinschwemmung im Horizont 4 in Verbindung mit der Aufweitung und Zerstörung der Glimmer beweisen einen längeren warmtemperierten Zeitabschnitt, in dem genügend elektrolytarmes Wasser zur Tonverlagerung zur Verfügung stand. Natürlich konnte die Tonverlagerung erst einsetzen, als durch die Entkalkung des Schotters ausreichend Ton für die Verwitterung freigesetzt worden war. Das brecciöse Gefüge, die Unversehrtheit der Toneinschwemmung in den Hohlräumen von Körnern im Zusammenhang mit den zu einzelnen Schichten aufgelösten Glimmern zu einem wirren Gefüge, kann als Anzeichen von Kryoklastik gedeutet werden (Altemüller, freundl. mündl. Mittlg.). Ableitend von diesen Befunden ist folgende Genese des fossilen Bodens zu skizzieren:

Sedimentation des kristallinarmen, sandigen Kieses (Horizont 6). Von oben nach unten fortschreitende Entkalkung, Freisetzung von Ton, danach und gleichzeitig Tonverlagerung, Aufbau von geschichteten Tonbelägen. Erosive Entfernung des A-Bodenhorizontes und Teile des $B_{t}$-Bodenhorizontes (Horizonte 3). Kryoturbate Bewegung und teilweise Verlagerung des Bodens in die unteren Bereiche des $B_{t}$-Bodenhorizontes reichend (Horizont 4). In einer kurzen Phase kam es zur erneuten Tonverlagerung, wodurch Klüfte mit geringmächtigen gut geschichteten Tonbelägen ausgekleidet wurden. Danach erfolgte die Ablagerung der hangenden Grundmoräne (Horizont 2) und der Boden wurde fossilisiert. Die dargestellten Befunde der mikromorpholgischen Untersuchung zeigen, daß die Verwitterungszone in Rottum-Wühlentobel (A 9) als ein echter fossiler Boden anzusehen ist. Der Boden befand sich an der ehemaligen Oberfläche und ist dort Verwitterungs- und Abtragungsvorgängen ausgesetzt gewesen und ist nicht innerhalb des Sedimentes durch Tiefenverwitterung entstanden.

Der fossile Boden in der $\mathrm{Kg} \mathrm{r}$. Riedw a nger ( A 7 ) ist heute leider nicht mehr zugänglich. Doch lassen die Beschreibungen von Graul (1962: 264) und SCHädel \& Werner (1963: 22) von einer Fließerde, die den fossilen Boden begleitet, darauf schließen, daß auch hier ein echter fossiler Boden entsprechend den Mindestbedingungen (HAAG 1979) vorlag. Nach den Geländebefunden ist auch der fossile Boden bei E h r e n s be r g (A 10 ) als ein echter fossiler Boden anzusprechen. Die erwähnten fossilen Böden beweisen einen warmzeitlichen Hiatus innerhalb der untersuchten Sedimente.

Eine solche Möglichkeit bieten einige Verwitterungserscheinungen in anderen Aufschlüssen nicht. Uber die Verwitterungszone in der Kgr. Fürstenwald bei Ochsenhausen (A 6) wurde schon früher berichtet (HAAG 1979). Ähnliche Verwitterungszonen befinden sich in der Kgr. südlich Hattenburg (A 5) und in der Kgr. nördlich Ringschnait (A 4). In ihnen liegen folgende Befunde für eine unvollständige Verwitterung vor: Gerölle mit karbonathaltigen Kernen, noch vorhandene Dolomitaschen, die Oberfläche der Verwitterungszone schwankt stark und zeigt Verbindungen zur heutigen Oberfläche. Die Geröllzusammensetzung ist im Liegenden und Hangenden der Verwitterung gleichartig. Es seien hier die mikromorphologischen Untersuchungen der Verwitterungszone in der $\mathrm{Kgr}$. nördlich Ringschnait am rechten Dürnachtalhang (A 4) angeführt. Im Aufschluß selbst konnte folgendes Profil der Verwitterungszone aufgenommen werden: 

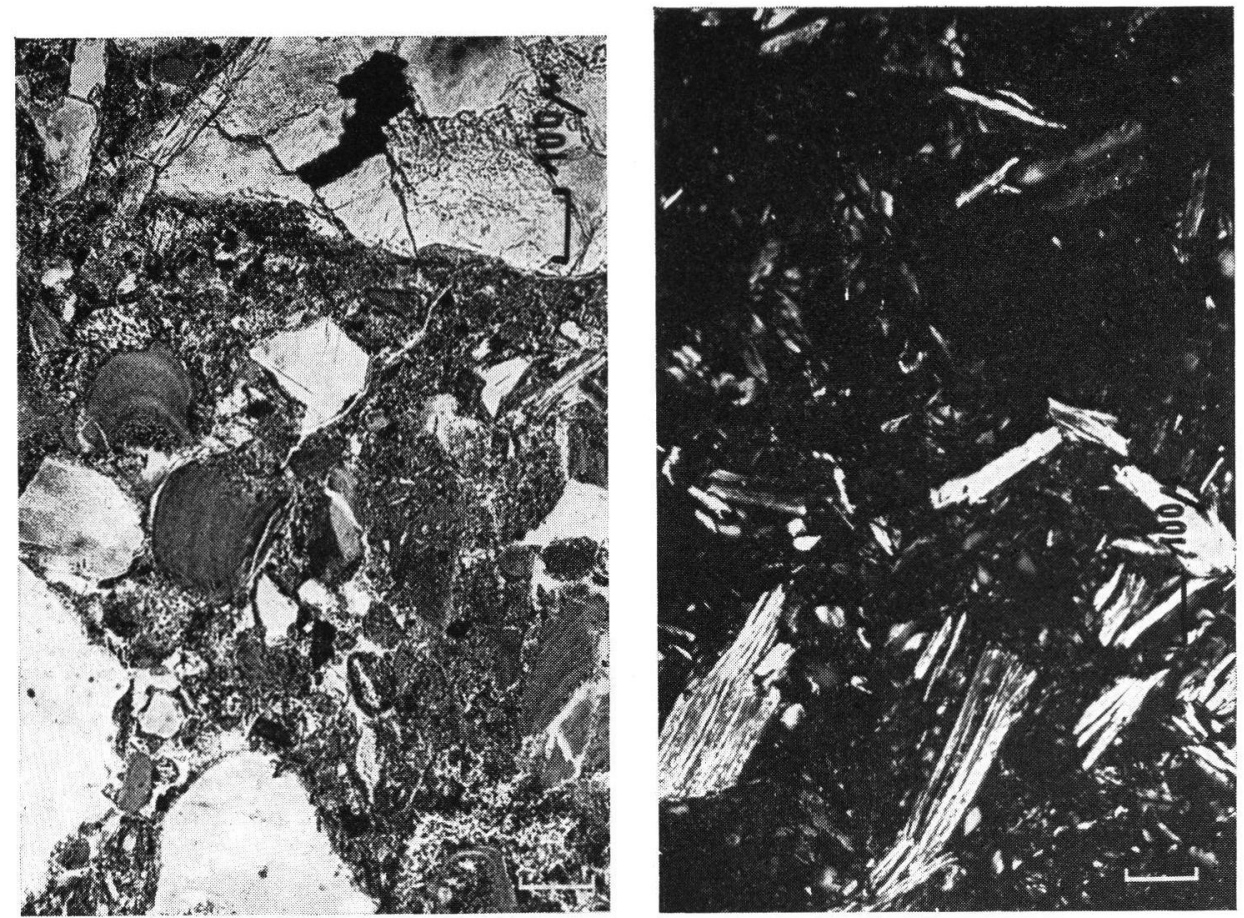

1

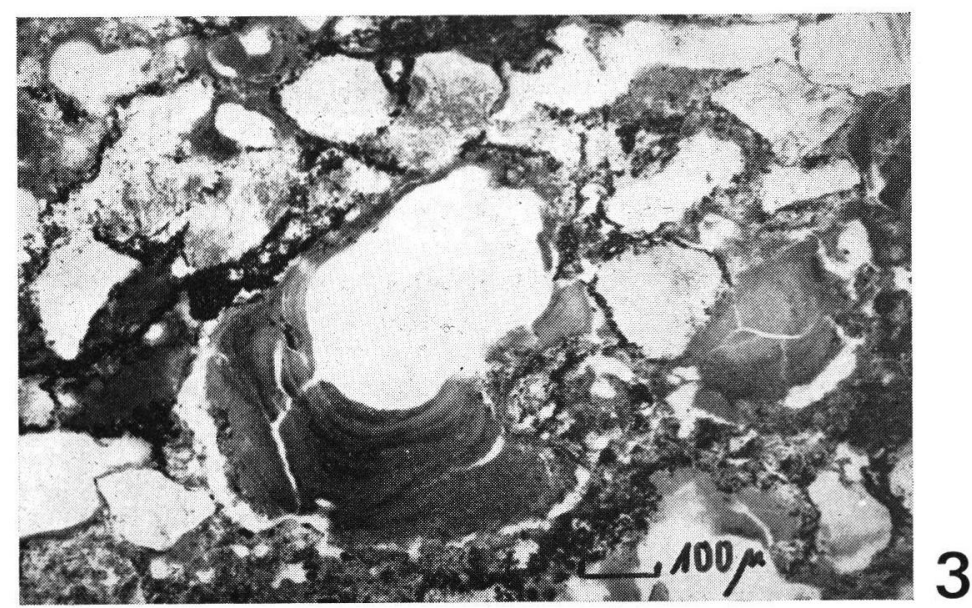

Tafel 1

Fig. 1: Rottum-Wühlentobel, brecciöses Gefüge im Horizont 4. Schollen von gut sortiertem, gut geschichtetem Ton schwimmen in einer unsortierten Grundmasse.

Fig. 2: Rottum-Wühlentobel, Biotite bei gekreuzten Polarisatoren. Die Biotite sind aufgeweitet und in Plättchen aufgelöst. In der rechten unteren Ecke ist eine Tonscholle an der leichten orientierten Doppelbrechung erkennbar, die in der Grundmasse schwimmt.

Fig. 3: Rottum-Wühlentobel, Hohlraumfüllung mit gut sortiertem, gut geschichteten Ton in einem Glaukonitsandstein. Im Gegensatz zu Bild 1 sind hier die Tonablagerungen nicht zerstört. Länge der Balken-Maßstäbe: $100 \mu$ 

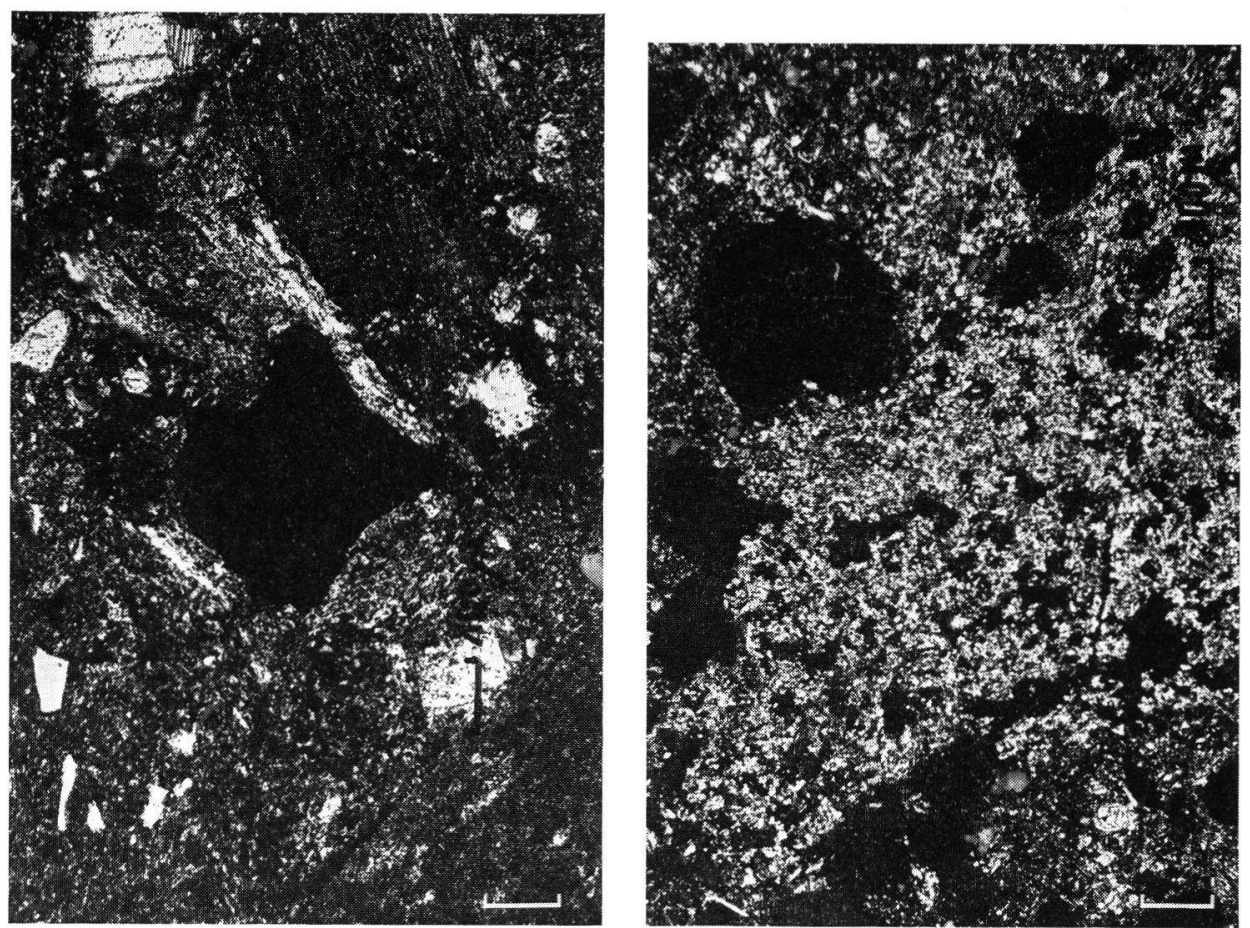

1

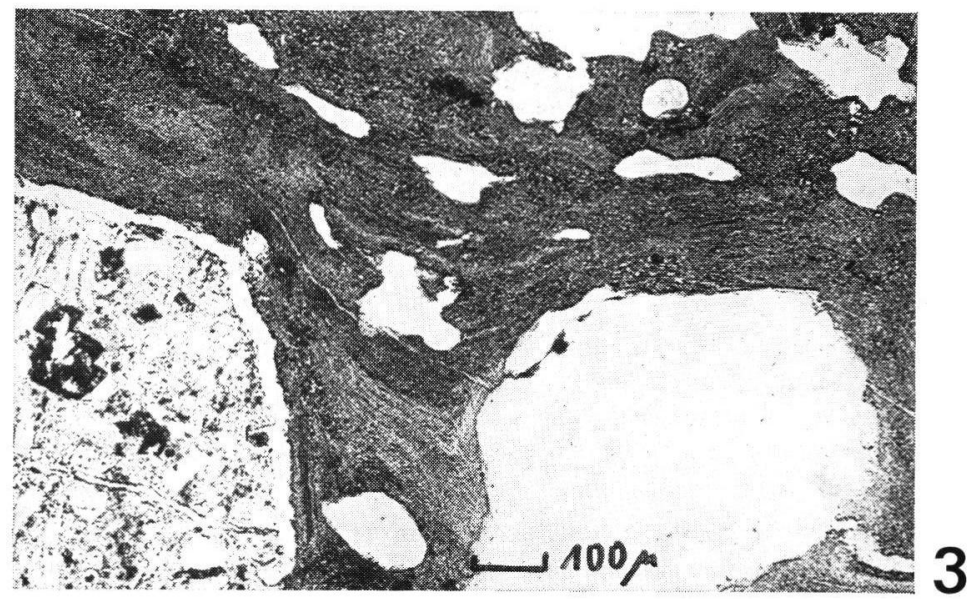

2

Tafel 2

Fig. 1: Kgr. Ringschnait, Hohlraum im Geschiebemergel des Horizontes 2 bei gekreuzten Polarisatoren. Die hellen länglichen Streifen an zwei Seiten des Hohlraumes sowie der kleinen Kluft nach rechts oben sind gut geschichtete Tonablagerungen. Die kleinen hellen Punkte stellen kleine Karbonatkristalle dar. Ebenso ist das Korn rechts oben ein Karbonat.

Fig. 2: Kgr. Ringschnait, Karbonatauflösung im Geschiebemergel von Horizont 2 bei gekreuzten Polarisatoren.

Fig. 3: Kgr. Ringschnait, Ablagerung von gut geschichtetem ungestörtem Ton zwischen Körnern von Horizont 4. Länge der Balken-Maßstäbe: $100 \mu$. 
1. $0-1,5 \mathrm{~m}$ brauner, $1 \mathrm{e} \mathrm{h} \mathrm{m}$ ig e $\mathrm{r} \mathrm{K}$ i e s, kalkfrei

2. 1,0-1,5 m grauer Geschiebe me rge 1, kalkhaltig, kristallinreich (s. Tab. 1, Probe Nr. 14)

3. $0,2-0,5 \mathrm{~m}$ brauner $1 \mathrm{e} \mathrm{h} \mathrm{mig}-\mathrm{s}$ andige $\mathrm{K} \mathrm{K}$ ies, kalkfrei, lagenweise mit grauem sandigem und kalkhaltigem Kies verzahnt, Kristallingehalt gleich Horizont 2

4. $1,0-1,9 \mathrm{~m}$ brauner $1 \mathrm{e} \mathrm{h} \mathrm{mig-s} \mathrm{a} \mathrm{n} \mathrm{dig} \mathrm{e} \mathrm{r} \mathrm{K}$ i e s, vereinzelt korrodierte Kalkgerölle und Dolomitaschen, nach unten taschenförmig in den kalkhaltigen Schotter greifend

5. 4,0 und mehr grauer, sandiger $\mathrm{K}$ i e $s$, kalkhaltig, im Kristallingehalt Horizont 2 entsprechend.

Taf. 2, Fig. 1 zeigt die Auskleidung eines Hohlraumes innerhalb des kalkhaltigen Geschiebemergels des Horizontes 2 mit Ton. Deutlich ist im polarisierten Licht die gut orientierte Doppelbrechung (hell) um den dunklen Hohlraum und die nach rechts oben anschließende Kluft zu sehen. Die hellen feinen Punkte der Grundmatrix sind die feinen Karbonatkristalle des Geschiebemergels. Es kommen Lösungserscheinungen sowohl an Karbonatgeröllen als auch der Grundmatrix vor (Taf. 2, Fig. 2). Manche der Hohlräume können auch mit feineren Karbonatkristallen als die umgebende Matrix ausgekleidet sein. An einer Stelle schwimmt ein Aggregat aus unveränderter Grundmoräne (Kartenhausstruktur der Biotite). Die Grenzen der aggregierten Grundmoräne wird von Schwundrissen nachgezeichnet. Biotite waren stellenweise an der Grenze des Grundmoränenaggregates zur umgebenden Matrix prallel zu dieser Grenze eingeregelt. Der Anteil an feinkristallinen Karbonatkörnern ist sehr hoch. In den unter dem Geschiebemergel folgenden Zonen 3 und 4 waren die Toneinschlämmungen in den oberen Bereichen unsortiert und ungeschichtet. Nach unten ging dieser unsortierte Ton allmälich in gut sortierten Ton mit guter Schichtung über (Taf. 2, Fig. 3). In den unteren Bereichen treten auch verstärkt korrodierte Karbonatkörner auf. Lösungshohlräume in Sandsteinen zeigen nur geringmächtige Auskleidung mit Tontapeten. Hier treten auch viele Körner mit einem karbonatischen Kern auf, während die äußere Schale entkalkt ist. Am unteren Ende von Horizont 4 nimmt der Karbonatgehalt allmählich $\mathrm{zu}$, einhergehend mit einem Rückgang der Korrosionserscheinungen an den Karbonaten.

Die von oben nach unten im Geschiebemergel zu verfolgende Toneinschlämmung zeigt, daß unter dem Geschiebemergel kein echter fossiler Boden vorliegt. Im Gegensatz zum Profil von Rottum-Wühlentobel fehlt die starke Zerstörung der Biotite und das brecciöse Gefüge. Diese Beobachtungen und die Geländebefunde aus Horizont 3 beweisen, $\mathrm{da}$ die Verwitterungszone durch Ti e f e n verwitte rung entstanden ist. Der Geschiebemergel scheint durch Frostvorgänge aufgelockert worden zu sein und war damit für Sickerwasser gut durchlässig. Eine Erklärung wäre ein nicht durch eine Verwitterungsdecke geschützter Geschiebemergel, der nach dem Eisrückzug bis zum erneuten Eisvorstoß über längere Zeit Frostvorgängen ausgesetzt war, ohne daß eine warmzeitliche vorangegangene Verwitterung eine schützende Decke geschaffen hatte. Es wären folgende Genese denkbar:

Ablagerung des Schotters von Horizont 3 bis 5 als Vorstoßschotter zum Geschiebemergel von Horizont 2. Vorrücken des Gletschers und Ablagerung des Geschiebemergels von Horizont 2. Rückzug des Eises und Freilegung des Geschiebemergels von Horizont 2 über längere Zeit bis zum erneuten Vorrücken des Gletschers. Auflockerung des über längere Zeit freiliegenden Geschiebemergels durch Frostvorgänge in der Zeit bis zur nächsten Warmzeit (es ist hierfür eine längere Zeit der Frosteinwirkung während des mittleren und jüngeren Riss anzusetzen. Danach Erwärmung und intensive Verwitterung während der Riß/Würm-Warmzeit). Bildung einer Verwitterungsdecke auf Geschiebemergel durch Entkalkung und Tonverlagerung. Die Tonverlagerung greift auf Klüften durch den aufgelockerten Geschiebemergel bis in den darunter befindlichen Schotter. Die Tonausfällung wird in dem Schotter durch zwei Faktoren begünstigt: einerseits geht die schnelle lineare 
Wasserbewegung auf den Klüften des Geschiebemergels in eine langsamere, mehr diffuse Wasserbewegung in den Hohlräumen des Schotters über; andererseits bildet sich an der Grenze von dem oben liegenden dichten Geschiebemergel (in Relation zum Schotter) zum darunter liegenden lockeren, durchlässigen Schotter aufgrund hängender Menisken ein Wasserstau aus (Fe-Ausfällung im Schliff). Der Prozeß der Tonverlagerung durch den Geschiebemergel in den darunter befindlichen Schotter dauert heute sicherlich noch an.

Alles spricht dafür, daß Tiefenverwitterung die Ursache der Verwitterungszone in der Kgr. Ringschnait (A4) ist. Die Verwitterungszone lag nie wie z. B. der fossile Boden von Rottum-Wühlentobel an der Oberfläche, sondern stellt eine von der heutigen Oberfläche gesteuerte Verwitterung dar und ist kein fossiler Boden.

\section{Bohrungen zur Bestimmung der Verwitterungstiefe}

In der jeweils letzten Zeile ist die D e u t u n g angegeben.

1. Klosterholz $2,5 \mathrm{~km}$ WNW Warthausen, $\mathrm{r} 56600$ h 33700

- 0,2 m Lehm, graubraun

- 4,6 m Lehm, braun, z. T. rot, etwas kiesig, kalkfrei

- 4,8 m Silt, graugelb, etwas kiesig, kalkig (Geschiebemergel)

$\mathrm{M}$ indel moräne

2. Windberg $1,5 \mathrm{~km} \mathrm{NW}$ Warthausen, r 58000 h 33960

- 0,3 m Lehm, hellbraun, feinsandig (Decklehm)

- 3,8 m Lehm, rotbraun, gleyfleckig, kiesig, kalkfrei

- 4,5 m Silt, gelbgrau, kiesig, kalkig (Geschiebemergel)

Mind e 1 mor äne

3. Mangoldsried 2,5 km W Warthausen, r 56690 h 33150

- 0,5 m Lehm, braun, kiesig, kalkfrei

- 1 m Kies, siltig, kalkig (kiesige Moräne)

Rißmoräne

4. Schrötersfeld 0,6 km NW Warthausen, r 58650 h 33180

-1 m Lehm, braun, kiesig, kalkfrei

- 1,2 m Kies, kalkig (kiesige Moräne)

$\mathrm{R}$ i ß mor än e

5. Herrschaftsholz $2 \mathrm{~km} \mathrm{NW}$ Laupertshausen, r 63160 h 33500

- 1 m Lehm, gelbbraun (Decklehm)

- 4,8 m Lehm, braun, gleyfleckig, tonig, wenig Kies, kalkfrei

Minde lmoräne

5'. Hungerberg, $P$ 574,7, $1 \mathrm{~km}$ W 5.

- 4,8 m Lehm, braun, kiesig, kalkfrei

Mindelmoräne

6. Romersbühl 1,4 km NNW Laupertshausen, r 63800 h 32950

- 1,6 m Lehm, braun, sandig-kiesig, kalkfrei

- 2,8 $\mathrm{m}$ Feinsand, lehmig, halbbraun, kalkfrei

- 5,8 m Lehm, braun und rötlichbraun, kiesig, kalkfrei

Mindelmoräne

7. Gurgel 1,6 km SW Laupertshausen, r 62800 h 31400

- 3,9 m Lehm, braungelb, tonig, sehr wenig Gerölle, kalkfrei

- 4,8 m Silt, grau, tonig, wenig Gerölle (Geschiebemergel)

Mindelmoräne 
8. Zum Stein, r 65700 h 31400

- 4,3 m Lehm, braun, tonig, wenig kiesig, kalkfrei

- 4,8 m Silt, graugelb, schwach kiesig, kalkig (Geschiebemergel)

Mindelmoräne

9. Binsenhau 2,2 km SE Laupertshausen, r 65430 h 29800

- 0,4 m Lehm, dunkelgrau, humos

- 4,8 m Lehm, braun, tonig, wenig kiesig, kalkfrei Mindelmoräne

10. $0,8 \mathrm{~km} \mathrm{NE}$ Winterreute, P. 627,9

- 2,7 m Lehm, braun, sandig-kiesig, kalkfrei

- 2,9 m Kies u. Sand, lehmig, kalkfrei

- 3,8 m Sand, kiesig, kalkig

kiesige Rißmor äne

11. Aspen NW Ringschnait, r 64960 h 27360

- 0,4 m Lehm, graubraun, humos

- 4,5 $\mathrm{m}$ Lehm, braun, sandig-kiesig, kalkfrei

- 4,6 m Kies-Sand, siltig, kalkig Minde $1 \mathrm{~m}$ or äne

12. Dicker Ghau 2,4 km NE Ringschnait, r 66720 h 28700

- 0,8 m Lehm, gelb, kalkfrei (Decklehm)

$-3 \mathrm{~m}$ Lehm, braun, kiesig, kalkfrei

- $4 \mathrm{~m}$ Lehm, gelb, kalkfrei (Decklehm?)

- 4,8 m Lehm, rotbraun, kiesig, kalkfrei

Nach geologischem Zusammenhang ist hier R i ß m or ä n e anzunehmen.

Die Verwitterungstiefe ist erhöht durch Fließerde und Decklehm, da der Bohrpunkt nıcht auf einer allseitig abfallenden Kuppe liegt.

13. Reinstetter Holz 2,8 km NE Ringschnait, r 67500 h 28660

- 0,3 m Lehm, dunkelbraun, humos

- 3,8 m Lehm, braun u. rot, sandig-kiesig, kalkfrei

- 6,3 m Lehm, gelbbraun, rotfleckig, kaum Gerölle, kalkfrei

- 7,8 m Lehm, rotbraun, stark sandig-kiesig, kalkfrei

$\mathrm{M}$ i nd e $1 \mathrm{~m}$ or $\ddot{\text { an e, kiesig }}$

14. E Ringschnait, $\mathrm{r} 66280$ h 26700

- $1 \mathrm{~m}$ Lehm, braun, sandig, kalkfrei

- 4,2 $\mathrm{m}$ Sand, fein, gelbbraun, kalkfrei (Flugsand)

- 5,2 $\mathrm{m}$ Sand, hellgrau, feinkiesig, kalkig (Flugsand)

- 5,8 $\mathrm{m}$ Sand, fein, braun, schwach kalkig

Flugsand auf $\mathrm{R}$ i $\beta$ morän e, die wenig westlich und in der alten Kiesgrube $0,5 \mathrm{~km}$ südlich (Aufschluß 10) mit 1,5 bis $2 \mathrm{~m}$ Verwitterungstiefe ansteht.

15. 1,4 km SE Ringschnait, P. 651,8

- 2 m Lehm, hellbraun, ohne Gerölle (Decklehm)

- 3,8 m Lehm, braun, wenig Kies, kalkfrei

Mindelmoräne

16. $1,5 \mathrm{~km}$ WNW Hattenburg, r 67920 h 25640

- $1 \mathrm{~m}$ Lehm, braun, stark gleyfleckig, einzelne Steinchen (Decklehm)

- 5,8 m Lehm, braun, schwach kiesig, kalkfrei

Minde 1 mor äne

17. $1,7 \mathrm{~km} \mathrm{SW}$ Hattenburg, r 67500 h 24550

P. 662,0

- 4,6 m Lehm, braun, wechselnd kiesig, kalkfrei

- 4,8 m Silt, graugelb, sandig-kiesig, kalkig

Mindelmoräne 
18. Mittelbuch SW-Rand, r 65500 h 23440

Graben und Bohrung

- $1 \mathrm{~m}$ Lehm, hellbraun, steinfrei (Decklehm)

- $4 \mathrm{~m}$ Lehm, braun, Geschiebe bis $40 \mathrm{~cm}$, kalkfrei

Ergebnis unsicher, da am flachen Hang, nach Lage: $R$ i B

19. $0,8 \mathrm{~km}$ SE Mittelbuch, P. 659,4

- $1 \mathrm{~m}$ Lehm, hellbraun, wenig Gerölle (Decklehm)

- 5,5 m Lehm, braun, feinsandig, kiesig, kalkfrei

- 5,8 m Silt, graugelb, feinsandig-tonig, kiesig, kalkig (Geschiebemergel)

Mindelmorän e

20. $0,9 \mathrm{~km}$ NNW Rottum, P. 656,5

- 1,1 m Lehm, braun, stark kiesig, kalkfrei

- 1,5 m Kies, kalkig (kiesige Moräne)

$\mathrm{R}$ iß mor äne

21. Höhe $0,8 \mathrm{~km} \mathrm{SW}$ Rottum, P. 665,4

-1 m Lehm, braun, wenig Kies

- 3,8 m Lehm, braun-rotbraun, kiesig, kalkfrei

Ungeeigneter Punkt, wahrscheinlich Fließerde auf Rißmoräne und spätere Muldenbildung westlich der Höhe

22. $1,7 \mathrm{~km}$ SE Steinhausen/Rottum, P. 703,0

- 4,6 m Lehm, braun, rostfleckig, kiesig, kalkfrei

- 4,8 m Silt, grau, schwach sandig-kiesig, kalkig

Minde lmorän e

23. $1,8 \mathrm{~km}$ E Füramoos, P. 679,1

- 1,8 m Lehm, hellbraun, wenig Kies (Decklehm)

- 4,8 m Lehm, braun, sandig-kiesig (unten stark) kalkfrei

Minde lmoräne

24. $2,2 \mathrm{~km} \mathrm{SE} \mathrm{Füramoos,} \mathrm{r} 68820$ h 17360

Seismikbohrung 1981. Quartäruntergrenze $650 \mathrm{~m}$

- $1 \mathrm{~m}$ Lehm, hellbraun, kaum Gerölle (Decklehm)

- $10 \mathrm{~m}$ Lehm, rotbraun, kiesig, kalkfrei ( $\mathrm{M}$ i n d e $1 \mathrm{~m}$ or ä n e )

- $13 \mathrm{~m}$ Lehm, rotbraun, wenig Gerölle, kalkfrei

- 17 m Kies, kalkig

- $33 \mathrm{~m}$ Kies mit Geschiebemergel, kalkig, etwa $16 \%$ Kristallin (Haslach?)

- 39 m Kies, kalkig, 1,3\% Kristallin (Zeiler Schotter, Günz)

- $44 \mathrm{~m}$ Sand, hellbraun, Glimmersand (OSM)

25. $1,9 \mathrm{~km}$ SE Ellwangen, P. 686,5

- 1,7 m Lehm, hellbraun, steinfrei (Decklehm)

- 6,0 m Lehm, braun-rotbraun, wenig kiesig, kalkfrei

Minde 1 moräne

26. Boschen $2,5 \mathrm{~km}$ NNE Hauerz, P. 691,4

- 5,8 m Lehm, braun, kiesig, kalkfrei

In benachbarter Seismikbohrung $7 \mathrm{~m}$ Verwitterungslehm auf Geschiebemergel.

Minde 1 mor äne

27. Unterpfauzenwald 3,5 km ESE Hauerz, r 75450 h 12700

Nach SChreiner \& Ebel 1981: 37

- 5-9 m Verwitterungs- und Decklehm, kalkfrei

- $15 \mathrm{~m}$ Geschiebemergel und Kies, kalkig

$\mathrm{M}$ indelmoräne 
28. Höhe W Steinhausen, P. 681,5

- 3,6 m Lehm, braun, kiesig, kalkfrei

- 3,8 m Kies, siltig, kalkig $\mathrm{M}$ in de l m or än e (Verwitterungstiefe vermutlich reduziert durch Bodenerosion)

28'. Steinhausen, P. 666,4

- 3,5 m Lehm, braun, schwach kiesig, kalkfrei

- 3,8 m Silt, graugelb, kiesig, kalkig

$\mathrm{M}$ in de $\mathrm{lm}$ or ä ne (Verwitterungstiefe vermutlich reduziert durch Bodenerosion)

20. W Englisweiler, P. 673,0

- 1,5 m Lehm, braun, kiesig, kalkfrei

- 1,8 m Silt, gelbgrau, kiesig, kalkig

Rißmoräne

30. S Englisweiler, P. 683,9

- 1,0 m Lehm, braun, sandig, steinig, kalkfrei

- 2,4 m Lehm, hellbraun, steinfrei, kalkfrei

- 2,8 m Silt, gelbgrau, kiesig, kalkig

Rißmoräne

31. $1,5 \mathrm{~km}$ SE Bellamont, P. 689,4

- 0,2 $\mathrm{m}$ humoser Waldboden, schwarz

- 0,4 m Lehm, gelb, steinfrei, kalkfrei (Decklehm)

- 4,8 m Lehm, braun, kiesig, kalkfrei

Minde lmor äne

\section{Aufschlußverzeichnis}

Nr.

top. Karte $1: 25000$

Koordinaten

$61650 / 34000$

A $1 \quad$ Kgr. Barabein

Bib N

$58900 / 30450$

A 2

Kgr. Scholterhaus

Bib N

$58900 / 30450$

A 3 Brückenbau Mettenberg

A $4 \quad$ Kgr. N Ringschnait

Och

$66250 / 28000$

A 5

Kgr. S Hattenburg

Och

69075 / 24600

A 6

Kgr. Fürstenwald

Och

$69500 / 24300$

A 7

Kgr. N Riedwanger

Och

$69100 / 22900$

A 8

Rottum Festplatz

Och

$69100 / 22375$

A 9

Rottum Wühlentobel

Och

$69200 / 22100$

A 10

Kgr. NE Ehrensberg

Och

$70200 / 21860$

A 11

Kgr. Mühlberg

Wur

$74000 / 17200$

A 12

Kgr. Häusern

Och

63675 / 25250

A 13

Kgr. Fürstenwald

P 628,3

Och

$70275 / 23400$

\section{Schriftenverzeichnis}

Altemüller, H.-J. (1960): Mikromorphologische Untersuchungen an einigen Gipskeuperböden im Raum Iphofen. - Bayer. Landw. Jahrb., 37, Sonderh. 4: 70-85; München.

- (1962): Verbesserungen der Einbettungs- und Schleiftechnik bei der Herstellung von Bodendünnschliffen mit VESTOPAL. - Z. Pflanzenern., Düngung, Bodenkde., 99: 164-177; Weinheim.

EichleR, H. (1970): Das präwürmzeitliche Pleistozän zwischen Riß und oberer Rottum. - Heidelberger geogr. Arb., 30: 128 S.; Heidelberg.

Fezer, F. (1969): Tiefenverwitterung circumalpiner Pleistozänschotter. - Heidelberger geogr. Arb., 24: 144 S.; Heidelberg. 
Graul, H. (1952): Geol. Karte $1: 25$ 000, Blatt Warthausen. - Mskr., Geol. Inst. Univ. Tübingen.

- (1962): Eine Revision der pleistozänen Stratigraphie des schwäbischen Alpenvorlandes (mit einem bodenkundlichen Beitrag von K. BrunnaCKER). - Peterm. Mitt., 106: 253-271; Gotha.

- (1968): Führer zur zweitägigen Exkursion im nördlichen Rheingletschergebiet, August 1968. - Heidelberger geogr. Arb., 20: 31-77, Heidelberg.

HAAG, T. (1979): Durch Oberflächeneinflüsse bedingte Entwicklung eines „fossilen Bodens“ im östlichen Rheingletschergebiet. - Jber. Mitt. oberrhein. geol. Ver., N.F., 61: 279-304, Stuttgart.

- (1981): Das Mindelglazial im östlichen Rheingletschergebiet zwischen Riß und Iller in Abgrenzung zur Günz- und Rißvereisung. - Diss. Univ. Gießen, 113 S.; Gießen.

- (1982): Das Mindelglazial zwischen Riß und Iller. - Jber. Mitt. oberrhein. geol. Ver., N.F., 64: 225-266, Stuttgart.

Kraus, K. (1930): Untersuchungen im Grenzgebiet und dem Vorland der größten Gletschervorstöße zwischen Biberach a. Riß und dem Bussen. - Jh. Ver. vaterl. Naturkde. Württemberg, 86: 1 -33, Stuttgart.

Löscher, M. (1976): Die präwürmzeitlichen Schotterablagerungen in der nördlichen Iller-LechPlatte. - Heidelberger geogr. Arb., 45: 157 S.; Heidelberg.

Mader, M. (1976): Schichtenfolge und Geschehensablauf im Bereich des Schussenlobus des pleistozänen Rhein-Vorlandgletschers. - Diss. Univ. Tübingen, 148 S.; Tübingen.

Penck, A. (1893): Bericht über die Exkursion des X. Deutschen Geographentages nach Oberschwaben und den Bodensee (10.-14. April 1893), - Verhandlg. X. deutsch. Geographentag zu Stuttgart, S. 216-223; Stuttgart.

- \& BRüCKNER, E. (1901/09): Die Alpen im Eiszeitalter. - 3 Bde., 1199 S.; Leipzig.

SCHÄdEL, K. \& Werner, J. (1963): Neue Gesichtspunkte zur Stratigraphie des älteren und mittleren Pleistozäns im Rheingletschergebiet. - Eiszeitalter u. Gegenwart, 14: 5-26; Ơhringen.

SCHREINER, A. (1980): Zur Quartärgeologie in der Umgebung des Eem-Interglazials von Krumbach/Saulgau (Baden-Württembrg). - Geol. Jb., A 56: 5-43, Hannover.

- \& Ebel, R. (1981): Quartärgeologische Untersuchungen in der Umgebung von Interglazialvorkommen im östlichen Rheingletschergebiet (Baden-Württemberg). - Geol. Jb., A 59: 65 S.; Hannover.

Weidenbach, F. (1937 a): Bildungsweise und Stratigraphie der diluvialen Ablagerungen Oberschwabens. - N. Jb. Miner. etc., Beil.-Bd., 78, Abt. B: 66-108; Stuttgart.

- (1937b): Erläuterungen zur geologischen Spezialkarte von Württemberg $1: 25$ 000, Bl. Biberach. - 91 S., mit geol. Kt.; Stuttgart.

- (1940): Erläuterungen zur geologischen Spezialkarte von Württemberg 1:25000, Bl. Ochsenhausen. - 61 S., mit geol. Kt.; Stuttgart. 
